Jonas Kuhn

\title{
Analysierte Parallelkorpora für die sprachvergleichende Grammatik: Was können computerlinguistische Methoden leisten?
}

\begin{abstract}
Dieser Beitrag versucht, eine Einschätzung der Einsatzmöglichkeiten für automatische Analysemethoden aus der aktuellen computerlinguistischen Forschung für die sprachvergleichende Grammatikforschung vorzunehmen. Zur Illustration werden die Ergebnisse einer computerlinguistischen Studie für die vergleichende Untersuchung von Spaltsatzkonstruktionen in verschiedenen Sprachen wiedergegeben und ausführlich diskutiert. Der Korpuszugang erfolgt in diesem Rahmen auf Basis einer vollautomatischen syntaktischen Analyse, die dann noch zusätzlich durch eine statistische Wortalignierung kontrastiv auf Parallelkorpora beleuchtet werden kann. Neben der Vorstellung der bereits bestehenden automatischen Annotationsmöglichkeiten, die in meinen Augen vielversprechende Wege für den sprachwissenschaftlichen Korpuszugang eröffnen, ist die Hoffnung, dass dieser Beitrag durch die abschließende Diskussion zu dem Bewusstsein beiträgt, dass eine tiefere, organischere Verbindung der beiden sprachwissenschaftlichen Disziplinen möglich ist: dann nämlich, wenn der Korpuszugang nicht mit statischen, vordefinierten Werkzeugen erfolgt, deren Verhalten durch die Grammatikforscherin oder den Grammatikforscher nicht beeinflusst werden kann, sondern wenn ein interaktiver Werkzeuggebrauch erfolgt, der von den vielfältigen Anpassungsmöglichkeiten mit den zugrunde liegenden maschinellen Lernverfahren Gebrauch macht.
\end{abstract}

\section{Einleitung}

Der wachsende Stellenwert von Korpusstudien für die empirische Untermauerung von sprachwissenschaftlichen Untersuchungen begründet einen großen Bedarf an geeignet annotierten Korpusdaten. Dort, wo der Untersuchungsgegenstand die Exploration von umfangreichen Datenmengen nötig macht - beispielsweise bei Untersuchungen zum Gebrauch von weniger häufigen syntaktischen Konstruktionen -, stellt sich die Frage, inwieweit automatische Analysewerkzeuge aus der computerlinguistischen Forschung einen stärkeren Beitrag zur Grammatikforschung leisten können, als dies bislang etabliert ist. Dieser Aufsatz ist der Versuch einer Einschätzung der gegenwärtigen Möglichkeiten und mittelfristigen Entwicklungsperspektiven in diesem Bereich - mit einem besonderen Schwerpunkt auf der sprachvergleichenden Forschung. 
Ich gehe von der Feststellung aus, dass die Computerlinguistik und die Sprachtechnologie in den letzten fünf bis zehn Jahren erhebliche Fortschritte erzielt haben - beispielsweise einerseits beim automatischen syntaktischen Parsen von Texten in einer Vielzahl von Sprachen und andererseits bei der Induktion von Wort-zu-Wort-Entsprechungen (einer so genannten Wort-Alignierung) über großen Sammlungen von übersetzten Texten. So lassen sich mit geringem manuellen Aufwand Parallelkorpora strukturell annotieren und sprachübergreifend alignieren. Dies kann eine große Chance für die sprachvergleichende Grammatikforschung sein.

Sicherlich ist noch zu klären, ob die mit automatischen Methoden derzeit erzielbare Qualität - die vom Blickpunkt vieler sprachtechnologischer Anwendungen bereits durchaus akzeptabel ist - für die Zwecke linguistisch anspruchsvoller Untersuchungen ausreicht. Führt man sich die seit einigen Jahren prinzipiell vorhandenen Möglichkeiten vor Augen, fällt jedoch in jedem Fall auf, dass sich in der Grammatikforschung ein entsprechender Werkzeugeinsatz noch nicht auf einer breiteren Basis etabliert hat. (Dies gilt nicht für die sehr etablierte Technik des Part-of-Speech-Tagging, also der automatischen Klassifikation der Wortformen im Korpus nach Wortarten und wichtigen morphologischen Merkmalen.)

Ich will in diesem Aufsatz nicht nur die Möglichkeiten des Einsatzes von computerlinguistischen Methoden illustrieren (dazu Abschnitt 3, nach einigen Bemerkungen zu den Qualitätsanforderungen an Korpusannotationen und zu ihrem wissenschaftlichen Status in Abschnitt 2), sondern ich versuche in Abschnitt 4 zudem, die eher zurückhaltende sprachwissenschaftliche Nutzung von automatischen Analysemöglichkeiten aus den Entwicklungen in der Computerlinguistik heraus zu rekonstruieren. Daraus werde ich in Abschnitt 5 ableiten, dass zwischen der Grammatikforschung (und gerade der sprachvergleichenden Grammatikforschung) und der Computerlinguistik in naher Zukunft ein sehr fruchtbares Zusammenwirken der unterschiedlichen Methoden der Sprachbetrachtung möglich ist - dass dafür allerdings wohl beide Seiten aufeinander zugehen müssen. Die Werkzeuge müssen möglicherweise für den Einsatz in der Grammatikforschung speziell vorbereitet werden, beispielsweise dürfte eine geeignete Darstellung der Ergebnisse die Nutzbarkeit erheblich erhöhen. Gleichzeitig ist für eine fundierte Interpretation der Ergebnisse beim computerlinguistischen Werkzeugeinsatz und für eine umfassende Ausnutzung der Möglichkeiten auf Seiten der Grammatikforschung ein Verständnis der zugrunde liegenden Mechanismen von erheblichem Vorteil. Eine laufende kompetente Anpassung der Analysewerkzeuge an die speziellen Anforderungen hätte die größten Erfolgschancen. Möglicherweise sollten zukünftig also die erforderlichen Kompetenzen gerade auch in der Ausbildung stärker berücksichtigt werden. 


\section{Zwischenbemerkung zur Korpusannotation: Qualitätsanforderungen und wissenschaftlicher Status}

Bevor in den späteren Abschnitten die Möglichkeiten eines computerlinguistischen Werkzeugeinsatzes diskutiert werden, soll an dieser Stelle ein mögliches Missverständnis aus dem Weg geräumt werden: Die automatische Analyse von Sprachdaten wird hier stets als Ergän₹ung eines Vorgehens verstanden, das sich vordringlich auf Korpusdaten stützt, die mit linguistischem Sachverstand und großer Sorgfalt von Hand annotiert wurden. In der sprachvergleichenden Grammatikforschung kann hierfür beispielsweise auf syntaktisch annotierte Korpora für verschiedene Sprachen zurückgegriffen werden, so genannte Baumbanken - hier eine beispielhafte Liste:

- Deutsch: TIGER Baumbank, ${ }^{1}$ NEGRA, ${ }^{2}$ TüBa-D $/ Z ;{ }^{3}$

- Englisch: Penn Treebank; ${ }^{4}$

- Niederländisch: Alpino-Treebank; ${ }^{5}$

- Schwedisch: Swedish Treebank; ${ }^{6}$

- Tschechisch: Prague Dependency Treebank. ${ }^{7}$

Für einen direkten Sprachvergleich liegen sogar handannotierte ParallelBaumbanken vor (allerdings in geringem Umfang, mit derzeit insgesamt 2.500 parallel annotierten Sätzen):

- SMULTRON: Stockholm Multilingual Treebank (Englisch/Deutsch/ Schwedisch, teilweise Französisch/Deutsch). ${ }^{8}$

Unter Annahme eines „konsensgesteuerten“ Konzepts von studienübergreifender Korpusannotation (das ich in Abschnitt 2.1 weiter erläutere) lassen sich die Ideal-Anforderungen für annotierte Korpusdaten in sprachwissenschaftlichen Untersuchungen folgendermaßen charakterisieren:

www.ims.uni-stuttgart.de/projekte/TIGER/; DFG-Projekt 1999-2004: Universität des Saarlandes, Universität Stuttgart und Universität Potsdam; ca. 900.000 laufende Wortformen (50.000 Sätze).

www.coli.uni-saarland.de/projects/sfb378/negra-corpus/; 355.096 Wortformen (20.602 Sätze). www.sfs.uni-tuebingen.de/tuebadz.shtml; 976.262 Wortformen (55.814 Sätze).

www.cis.upenn.edu/ treebank/; Wall Street Journal-Artikel (1 Mio. Wortformen) und andere Texte.

www.let.rug.nl/ vannoord/trees/.

http://w3.msi.vxu.se/ nivre/research/st.html.

http://ufal.mff.cuni.cz/pdt/.

www.cl.uzh.ch/research/paralleltreebanks/smultron.html; Department of Linguistics, Stockholm University und Institut für Computerlinguistik, Universität Zürich. 
1) Für den Sprachausschnitt der jeweiligen linguistischen Untersuchung sollte eine ausreichend große repräsentative Stichprobe von Daten vorliegen;

2) die Annotation sollte nicht auf Annahmen basieren, die in der eigenen Studie nicht geteilt werden;

3) die Annotation sollte 100\% fehlerfrei sein.

Bei der Konstruktion von Baumbanken für eine studienübergreifende Nutzung wird in der Regel versucht, sich diesen Ideal-Anforderungen anzunähern und

1') eine relativ große Sammlung von typischen Daten zu annotieren,

2') eine weitgehend theorie-neutrale Annotation zu verwenden und

3') anhand von sorgfältigen Richtlinien und durch Qualitätskontrolle eine systematische, flächendeckende Annotation mit geringer Fehlerquote bereitzustellen.

Dennoch kann man bei Korpusuntersuchungen zu Fragen der Grammatikforschung schnell an die Grenzen der vorhandenen Baumbanken stoßen. Für die Untersuchung von Interaktionen zwischen mehreren Phänomenen ist die Stichprobe häufig zu klein und/oder der Sprachausschnitt entspricht nicht den Anforderungen; im ungünstigen Fall enthält das in der Baumbank gewählte Annotationsformat eine für die eigene Studie wesentliche Unterscheidung nicht; und trotz Qualitätskontrolle ist bei seltenen Phänomenen eine inkonsistente Behandlung in Baumbanken praktisch nicht zu vermeiden, wie die Erfahrung mit den bestehenden, hochwertigen Baumbanken lehrt.

Daher ist es günstig, wenn die Arbeit mit sorgfältig annotierten Korpora durch ein automatisches Verfahren mit einer hinreichenden Verlässlichkeit ergänzt werden kann - das in der Anwendung selbstverständlich entsprechend relativiert werden muss (beispielsweise durch nachträgliche manuelle Sichtung).

\subsection{Exkurs: Annahmen zum Status von Korpusannotation}

An dieser Stelle ist es günstig, ein wenig zu präzisieren, welches die Grundannahmen bzw. Arbeitshypothesen zum wissenschaftlichen Status von Korpusannotationen sind, von denen dieser Beitrag ausgeht (und die wohl den meisten Anstrengungen, aus denen die oben genannten handannotierten Ressourcen hervorgegangen sind, mindestens implizit zugrunde liegen). ${ }^{9}$ Leser,

Ich danke einer Person unter den anonymen Gutachtern dafür, dass er oder sie die Klärung dieser gerade in computerlinguistischen Arbeiten häufig impliziten Annahmen angemahnt hat. Sicherlich bleibt der Beitrag geprägt von den Grundannahmen aus dem Arbeitsumfeld des Autors (also der Computerlinguistik und der computerlinguistisch geprägten generativen Grammatiktheorie) - eine adäquate Würdigung unterschiedlicher korpuslinguistischer Über- 
die zunächst ein Gefühl für die Möglichkeiten des Werkzeugeinsatzes entwickeln wollen, mögen den Rest dieses Teilabschnitts bei der ersten Lektüre eventuell überspringen.

Generell ist in empirischen Wissenschaften zwischen Beobachtungen und deren Interpretation zu unterscheiden, und es ist für ein nicht-zirkuläres wissenschaftliches Vorgehen von zentraler Bedeutung, beides nicht zu vermischen. Was heißt das für den Status von Korpusdaten und deren Annotation in der Grammatikforschung? Eine vorsichtige Position, die man „annotationsskeptisch“ nennen könnte, verweist darauf, dass jede Annotation eines Korpusbelegs eine Interpretation der empirischen Beobachtung (nämlich der reinen Sprachdaten) durch die Wissenschaftlerin oder den Wissenschaftler ist. Somit muss jede Verwendung eines bereits annotierten Korpus für eine neue Studie mit großer Vorsicht betrachtet werden: Aus den Annotationen (= Interpretationen) könnten Fehlschlüsse hinsichtlich der zugrundeliegenden Beobachtungen gezogen werden, auf die ja nicht mehr direkt zugegriffen wird. Gerade für die vergleichende Grammatikforschung ist diese Vorsicht gelegentlich sicherlich sehr angebracht: Je nach der deskriptiven Tradition oder der theoretischen Schule, in der eine vorgefundene grammatische Korpusannotation steht, mag sie einen funktionellen (oder formalen) Parallelismus mit einer Kontrastsprache verschleiern oder ungerechtfertigter Weise suggerieren. ${ }^{10}$

Dem gegenüber steht eine verbreitete Position zur studienübergreifenden Korpusannotation, die man vielleicht mit „Annotation des deskriptiven Konsens" umschreiben könnte, und die davon ausgeht, dass sich die komplexeren sprachwissenschaftlichen Interpretationen von empirischen Beobachtungen in Korpusdaten in der Regel aus vielen kanonischen Bausteinen der Teilinterpretation zusammensetzen. Für diese unstrittigen Bausteine einer abstrakteren Interpretation ist eine Wiederverwertung ausgesprochen ökonomisch. Beispielsweise betreffen lokal ambige Flexionsformen (wie Kasussynkretismen) oder Anbindungsambiguitäten von Präpositionalergänzungen eine Ebene der grammatischen Beschreibung, die nicht von speziellen theoretischen Annahmen abhängt, und sie lassen sich in den allermeisten Fällen

zeugungen kann hier nicht geleistet werden. Es sei daher darauf verwiesen, dass es in der Fachdiskussion der letzten Jahre eine Zahl von Beiträgen gibt, die sich mit dem Status von Annotation und insbesondere mit Annotationsfehlern auseinandersetzen, z.B. Giesbrecht/ Evert (2009); Meurers/Müller (2009); Belica et al. (2011).

10 Ein bekanntes Beispiel betrifft den Status von pronominalen Subjekten im modernen Französisch als Klitika bzw. Kongruenz-Marker, zu der es beispielsweise in der generativen Literatur unzählige Diskussionsbeiträge gibt, ausgehend von Kayne (1975). Würde nun eine gezielte Vergleichsstudie zu Klitika unreflektiert eine naive bestehende Korpusannotation französischer Subjekte als eigenständige Pronomina übernehmen, könnte dies zu Fehlschlüssen führen. Andere Beispiele sind typologisch stark von den indoeuropäischen Sprachen abweichende grammatische Systeme, bei denen Korpusannotationen (je nach Herkunft) möglicherweise von einer anglozentrischen Sichtweise geprägt sein können. 
aus dem Kontext heraus durch Muttersprachler zweifelsfrei auflösen. Mit einem sorgfältigen Annotationsansatz können sie also einer Vielzahl von darauf aufbauenden Studien zugänglich gemacht werden, für die man sich im Zuge der wissenschaftlichen Interpretation ohnehin auf die kanonische Kategorisierung berufen würde (beispielsweise müsste jede Studie zu Prinzipien der Wortstellung ohne Zugriff auf Vorabannotationen en passant die Desambiguierung von Kasusformen vornehmen, gegebenenfalls immer wieder für die gleichen Korpusdaten); und so erweitern studienübergreifende Annotationen die in einem gegebenen Zeitrahmen verwertbare empirische Basis ganz erheblich. (Ähnlich erfolgt der Austausch in anderen empirischen Wissenschaften wie z.B. der Astrophysik nicht ausschließlich auf Basis von Primärdaten der experimentellen Beobachtung, sondern unter Einbeziehung von kanonischen Modellierungen auf einer abstrakteren Ebene. ${ }^{11}$

Für den sprachwissenschaftlichen Umgang mit Korpora setzt das Vorgehen selbstverständlich voraus, dass die vorgefundenen Annotationen in reflektierter Form eingesetzt werden. So kann eine im Allgemeinen unbedenkliche kontext-basiert desambiguierte Kasusannotation nicht die ausschließliche Grundlage für eine Studie sein, die explizit die Grenzbereiche einer bestimmten kanonischen Kategorisierung zum Forschungsinhalt hat (beispielsweise die Verwendung von synkretistischen Formen in freien Relativsätzen wie die Nominativ/Akkusativ-Form was in Karla hat gegessen, was übrig war). ${ }^{12}$ Die Annahmen, die hinter einer Annotation stehen, müssen dokumentiert sein und diese Dokumentation muss bei der Verwendung in Betracht gezogen werden.

Das Maß an Konsensfähigkeit einer Kategorisierung lässt sich zudem empirisch untersuchen und statistisch quantifizieren, indem die Übereinstimmung zwischen den Annotationsergebnissen mehrerer Annotatoren auf denselben Ausgangsdaten (das inter-annotator agreement) gemessen wird. Schließlich kann und sollte - wie in anderen empirischen Wissenschaften - bei der quantitativen Analyse von Frequenzverteilungen in Korpusdaten, die auf bestimmten Annotationsentscheidungen oder komplexeren Filtern aufsetzen (oder die aus anderen Gründen, wie der Verwendung von automatischen Werkzeugen, als partiell fehlerbehaftet bekannt sind), eine Signifikanz- bzw. Fehlerabschätzung erfolgen.

Die zuletzt geschilderte Position einer „konsensgesteuerten“ Annotation kann als Ausgangspunkt für die weitergehenden Betrachtungen in diesem Beitrag gesehen werden, die eine Erweiterung der relativ verlässlichen manuellen Konsensannotation mit automatischen Mitteln in Erwägung zieht. Sofern ein reflektiertes Vorgehen unter Einbeziehung von Fehlerabschät-

$11 \quad$ Streng genommen beinhaltet ein derartiges Vorgehen immer das Risiko der Fortpflanzung eines Interpretationsfehlers, andererseits wäre der breitere wissenschaftliche Fortschritt ohne kanonische Abstraktionen stark eingeschränkt.

12 Und dennoch ist auch hier denkbar, dass die kanonische Annotation für das Auffinden der relevanten Beobachtungen hilfreich ist. 
zungen gewählt wird, bedeutet der Schritt keinen grundlegenden methodologischen Wandel: Es werden lediglich ,riskantere“ (automatische) Annäherungen an einen deskriptiven Konsens bereitgestellt, für die das Fehlerrisiko gegen den potenziell erheblichen Nutzen durch die schnelle Erschließung von wesentlich größeren Datenmengen abgewogen werden muss.

Neben dieser auf Intersubjektivität der Konsensannotation (= Interpretation) abhebenden Motivation für die Erzeugung von studienübergreifenden Annotationen der zugrundeliegenden reinen Korpusdaten (= Beobachtungen) gibt es eine alternative Sichtweise auf den wissenschaftlichen Status von annotierten Korpora. Diese sei hier noch kurz skizziert, da sie sich, zumindest implizit, im Selbstverständnis vieler informatiknaher Arbeiten zur Sprachverarbeitung bzw. zur Künstlichen Intelligenz widerspiegelt, die annotierte Korpora als Datengrundlage für maschinelle Lernverfahren einsetzen. Zunächst sind Text- und Sprachkorpora offensichtlich Sammlungen von Sprachproduktionsdaten. Die Annotation von Beschreibungskategorien und -relationen ist somit Teil der wissenschaftlichen Interpretation; sie unterliegt der wissenschaftlichen Theoriebildung und darf unter diesem Blickwinkel nicht mit empirischen Beobachtungen vermischt werden. Andererseits können sprachliche Einheiten aus den Produktionsdaten verwendet werden, um kontrollierte Experimente zum Sprachverstehen durchzuführen. So kann die syntaktische Annotation eines Satzes im gegebenen Korpuskontext als Experiment verstanden werden, bei dem der Annotator als Versuchsperson aus einer Vielzahl von möglichen kognitiven Interpretationen eine auswählt und diese anschließend in die zugehörige strukturelle Analyse übersetzt. Somit wird die im Kopf des Annotators entstandene kognitive Repräsentation Ziel einer indirekten empirischen Beobachtung, die mangels einer direkten Aufzeichnungsmöglichkeit in eine symbolische Teilrepräsentation rückübersetzt werden muss. In vergleichbarer Weise werden Daten zu anderen kognitiven Fähigkeiten des Menschen relativ zu einem Korpus von „Eingabedaten“ erhoben und aufgezeichnet (zu visuellen Fähigkeiten beispielsweise eine sprachliche Benennung der erkannten Gegenstände oder Personen relativ zu gezeigten Bildern).

Natürlich ist die indirekte Aufzeichnung von Beobachtungen im Falle der Verwendung von linguistischen Repräsentationen eher angreifbar, insofern als sie in der Regel eine gewisse theoretische Schulung (also mögliche Beeinflussung) der Versuchsperson voraussetzt; bei sorgfältig ausgearbeiteten Annotationsrichtlinien lässt sich die Tragweite dieser Problematik jedoch in aller Regel recht klar einschränken. ${ }^{13}$ Das oben genannte Maß des inter-annotator agreement erhält unter dieser alternativen Sichtweise einen an-

Es gibt Vorschläge zu einer indirekten strukturellen Korpusannotation, die das Problem der Theorieabhängigkeit bestimmter syntaktischer Repräsentationen dadurch umgeht, dass die Annotationsaufgabe in die Beurteilung möglicher Implikationen des Korpusbelegs verlagert wird (Yuret et al. 2010): Impliziert beispielsweise der Satz The man with the hat was tired die Aussage The man was tired oder die Aussage The hat was tired? 
deren Status: Es bewertet nicht mehr den Konsens hinsichtlich einer kanonischen wissenschaftlichen (Teil-)Interpretation, sondern es bewertet die Bandbreite der beobachtbaren kognitiven Entscheidungen bzw. Ausgaben, die bei der gegebenen Eingabe möglich sind (sofern man idealisierend davon ausgeht, dass Abweichungen nicht in dem verbleibenden „Rückübersetzungsschritt" eingeführt werden).

Die Rekonstruktion eines annotierten Korpus als Menge von Paaren von empirisch beobachteten Ein- und Ausgaben kann in der Tat den Einsatz von maschinellen Lernverfahren für die Simulation bzw. Modellierung von kognitiven Prozessen (wie Teilprozessen des Sprachverstehens) motivieren. Die aus Sicht der (vergleichenden) Grammatikforschung zentralen wissenschaftlichen Fragestellungen werden wohl durch das zuvor skizzierte „konsensgesteuerte“ Annotationskonzept unmittelbarer abgebildet - um ein Verständnis für die Vorgehensweise beim Einsatz von maschinellen Lernverfahren zu entwickeln, ist es jedoch sicherlich sinnvoll, sich auch die alternative Konstruktion vor Augen zu führen.

Für beide Sichtweisen gilt gleichermaßen, dass ein reflektiertes und selbstkritisches Vorgehen beim Einsatz der Annotationen angebracht ist.

\section{Illustration der computerlinguistischen Möglichkeiten: Identifikation von Spaltsätzen}

In diesem Abschnitt soll beispielhaft illustriert werden, in welcher Weise die automatische Strukturanalyse (insbesondere von Parallelkorpora) die Arbeit mit Korpusdaten in der vergleichenden Grammatikforschung unterstützen kann. Ein dankbares Beispiel hierfür ist das Auffinden von Instanzen von Spaltsätzen wie in (1) und (2) in unterschiedlichen Sprachen im Europarl-Parallelkorpus, das aus den Protokollen der Debatten des Europäischen Parlaments in 11 (bzw. für die Jahre seit 2007 21) Sprachen besteht (Koehn 2005). Zu dieser Suchaufgabe haben wir in Bouma et al. (2010) für die entsprechenden Konstruktionen im Deutschen, Niederländischen, Englischen und Schwedischen methodologische Experimente mit unterschiedlichen Suchstrategien durchgeführt. Die eckigen Klammern deuten die vier Bestandteile an, die zur strukturellen Identifikation der Spaltsatzkonstruktion erkannt werden müssen: das Expletivpronomen, die Kopula, die hervorgehobene Konstituente und ein Relativsatz bzw. Komplementsatz.

(1) [Es] [sind] [die einfachen Menschen], [die leiden].

(2) [It] [is] [precisely upon price levels] [that consumer policy has failed to focus sufficiently]. 
Die Datengrundlage für unsere Experimente waren die jeweiligen Sprachversionen des Europarl-Korpus mit ca. 1,5 Millionen Sätzen je Sprache. Mit dieser Größe ist das Korpus eine sehr geeignete Grundlage für grammatische Untersuchungen. Aufgrund der großen Datenmenge und der relativen Seltenheit der Spaltsatzkonstruktion scheidet eine rein manuelle oder rein auf Wortformen basierende Suche aus. Zum Vergleich der Analysemöglichkeiten haben wir die Verwendung von klassischen regulären Suchausdrücken auf der Wortfolge (einschließlich einer automatischen Wortartenbestimmung) auf der einen Seite mit der Verwendung von vollautomatischen Parsern für die vier Sprachen auf der anderen Seite verglichen. Ich will das Vorgehen und einige wichtige Resultate in den folgenden beiden Abschnitten ausführlich erläutern, da es das Spannungsfeld der analytischen Möglichkeiten gut verdeutlicht.

Auf den kontrastiven Aspekt, der sich aus der Verwendung von Parallelkorpora ergibt, gehe ich in Abschnitt 3.3 noch gezielt ein. Generell sollen die Untersuchungen hier nur die technischen Möglichkeiten einer (halb-)automatischen Suche illustrieren; für eine wesentlich tiefer gehende vergleichende Betrachtung sei beispielsweise auf Gast/Wiechmann (in diesem Band) verwiesen, die für das Sprachpaar Deutsch/Englisch die Verwendung von $w(h)-$ Clefts im Europarl-Korpus vergleichen.

\subsection{Spaltsatzsuche mit regulären Ausdrücken}

Nach dem klassischen Suchverfahren - ohne direkten Zugriff auf die syntaktische Struktur des Korpusmaterials - wird man versuchen, einen Suchausdruck über Wortfolgen zu formulieren, der allgemein genug ist, dass er nicht nur auf einen Subtypus der gesuchten Konstruktion zutrifft, der aber gleichzeitig präzise genug ist, dass er zu einer handhabbaren Menge von Treffern führt, die dann manuell durchsucht werden müssen, um die tatsächlichen Instanzen herauszufiltern.

In (3) ist ein typischer Suchausdruck angedeutet, der diesen beiden Kriterien gerecht zu werden versucht. Ein wesentlich umfassenderer, aber von der grundsätzlichen Strategie her vergleichbarer Ausdruck wird beispielsweise von Dufter (2009) angesetzt und im Rahmen der Anfrageschnittstelle zum Europarl-Korpus des OPUS-Korpus verwendet (http://opus.lingfil.uu.se/; Tiedemann/Nygaard 2004).

$$
\begin{aligned}
& \text { „Es“" „ist } \mid \text { sind } \mid \text { war } \mid \text { waren“ [KEIN-KOMMA]+ „," „der } \mid \text { die } \mid \text { das } \mid \\
& \text { den } \mid . . . “
\end{aligned}
$$

Die Formulierung „[KEIN-KOMMA]+“ soll andeuten, dass ein oder mehrere Tokens (also Wortformen oder Interpunktionszeichen) stehen können, die jedoch keine Kommata sein dürfen. Es ist naheliegend für Spaltsatz- 
Kandidaten anzunehmen, dass sich die Wortfolge durch eine relativsatzähnliche Konstruktion auszeichnet, die im Anschluss an die Folge „Es ist/sind/ war/waren ..." anschließt, ohne dass weitere Satzeinbettungen intervenieren - was sich durch den Ausschluss von Kommata gut annähern lässt.

Beispiel (4) zeigt jedoch eine Spaltsatzinstanz aus dem Europarl-Korpus, die auf diesem Weg fälschlicherweise ausgeschlossen wird (man spricht von einem so genannten falsch negativen Befund oder einem Fehler des Typs II). Denn natürlich kann die im Spaltsatz hervorgehobene Konstituente selbst einen Attributsatz enthalten, wenn dies auch selten der Fall ist.

[Es] [ist] [die Tatsache, daß Menschenopfer zu beklagen sind], [die diese Katastrophe aus europäischer Sicht so außergewöhnlich macht].

Auf Grundlage der linearen Folge von Wortformen und Satzzeichen lassen sich diese seltenen Spaltsatzinstanzen allerdings praktisch nicht automatisch von sehr häufigen Satzgefügen unterscheiden, in denen der Relativsatz an einer anderen Stelle angebunden ist, wie beispielsweise in (5). Derartige Beispiele würden ohne einen Anschluss von intervenierenden Kommata also als so genannte falsch positive Befunde (oder Fehler des Typs I) ausgegeben.

(5) Es ist bekannt, daß Personen, die sich draußen aufhalten, dem größten Risiko ausgesetzt sind [...]

Das Sichten von Kandidatenlisten würde damit erheblich erschwert, so dass man in vielen Fällen stattdessen in Kauf nehmen wird, dass falsch negative Befunde vom Typ (4) verloren gehen.

Solange die übrigen Eigenschaften der „versäumten“ falsch negativen Befunde völlig unabhängig vom Gegenstand der linguistischen Untersuchungen sind, auf den die Korpusstudie abzielt, ist gegen ein derartiges Vorgehen nicht viel einzuwenden - die Verteilung der untersuchten Eigenschaften sollte dann auch innerhalb der eingeschränkten Menge von aufgefundenen Instanzen repräsentativ sein. Im allgemeinen Fall wird man jedoch nicht a priori ausschließen können, dass das Ausklammern einiger strukturell schwer zu identifizierender Instanzen nicht doch zu einer Verzerrung der vorgefundenen Eigenschaften führt. ${ }^{14}$ So ergibt sich die Gefahr

$14 \quad$ Ein noch größeres Problem als das bewusste Ausklammern von schwer zu identifizierenden Strukturen liegt (unabhängig von der Analysetiefe) generell darin, dass man während der Optimierung von Suchausdrücken nur die falsch positiven Befunde tatsächlich zu Gesicht bekommt (beim Sichten der Ergebnislisten). Die Gefahr, völlig unbewusst eine wichtige Klasse von Befunden ausgeschlossen zu haben - also mit falsch negativen Befunden leben zu müssen - ist dadurch stets präsent. Im Rahmen dieses Beitrags kann auf diese Problematik nicht ausführlich eingegangen werden. Sie macht gezielt eingesetzte unabhängige Tests auf mögliche falsch negative Befunde erforderlich. Dabei kann die parallele Verwendung von unterschiedlichen automatischen Analysewerkzeugen für ein heuristisches Vorgehen hilfreich sein - wobei Sorge zu tragen ist, dass zwischen den Werkzeugen auch keine schädliche versteckte Abhängigkeit besteht (wenn beispielsweise Teilkomponenten des Werkzeugs aus den gleichen Entwicklungs- oder Trainingsdaten erzeugt wurden). 
von möglichen Fehlschlüssen folgender Art: In Sprache 1 finden sich in einer durch Korpussuche ermittelten Stichprobe der Konstruktion A (z.B. Spaltsätze) deutlich weniger Belege mit der Eigenschaft X (z.B. Indefinitheit der hervorgehobenen Konstituente) als in Sprache $2-$ also unterscheiden sich die beiden Sprachen systematisch in der Verwendung der Konstruktion A. Hier kann eben nicht ausgeschlossen werden, dass die Eigenschaft X lediglich aufgrund der Vereinfachung des Suchausdrucks in der Stichprobe unterrepräsentiert ist.

Zwei weitere deutsche Spaltsatzinstanzen, die der naheliegende Ausdruck in (3) ,übersieht“, sind in (6) und (7) aufgelistet:

(6) Die russischen Behörden stellen es nicht mehr bereit, somit [sind] [es] [die Nichtregierungsorganisationen], [die sich darum kümmern].

(7) [Es] [sind] [die jungen Menschen], [die abwandern], [diejenigen], [die Ausbildung und Beschäftigung außerhalb der ländlichen Gebiete finden], was sich in diesen Regionen sehr ungünstig auswirkt.

Die durch (6) illustrierten Stellungsvarianten (das Expletivpronomen steht hier nach der Kopula, da das Vorfeld durch somit besetzt ist) werden zwar jeder Betrachterin und jedem Betrachter der deutschen Grammatik bewusst sein - dass man jedoch bei der Formulierung von Suchausdrücken zu jedem Zeitpunkt das gesamte Stellungsspektrum im Auge behalten kann, ist nicht realistisch. Und gerade im Zusammenhang mit den Stellungsvarianten sind Korrelationen mit bestimmten informationsstrukturellen Eigenschaften der Elemente in der Konstruktion geradezu zu erwarten - also dürfte eine gewisse Verzerrung der Stichprobe vorprogrammiert sein.

Beispiel (7) zeigt eine sicherlich recht seltene (konjunktionslose) Koordinationskonfiguration, deren zweites Konjunkt (diejenigen, die ... finden) durch einen regulären Suchausdruck schwer zu identifizieren ist.

\subsection{Spaltsatzsuche auf geparsten Texten}

Für den Vergleich der klassischen Suche mit einem computerlinguistisch besser informierten Ansatz setzten wir in Bouma et al. (2010) robuste Dependenzparser ein, so dass wir für die Spaltsatzsuche eine automatische syntaktische Analyse der Korpussätze verwenden konnten. Dieser Abschnitt fasst das Vorgehen und die Evaluation im Vergleich mit regulären Ausdrücken zusammen.

\subsubsection{Automatisches Dependenzparsing für die Spaltsatzsuche}

Abbildung 1 zeigt eine rudimentäre grafische Darstellung der Dependenzstruktur für Satz (1), wie wir sie im Rahmen der Entwicklung der Suchaus- 
drücke verwendeten. ${ }^{15}$ Mit einer horizontalen Linie verbunden sind jeweils ein Kopfwort (markiert mit „ „H“) und seine Dependenten, also die von ihm abhängigen Wörter (markiert mit den Namen der Abhängigkeitsrelation: „sb“ für Subjekt, „pd“ für Prädikativ, „rc“ für Relativsatz, „,nk“ für eine unterspezifizierte Relation zwischen dem Kopf einer Nominalphrase und weiteren Elementen der „Kern-NP“, „punc“ für Satzzeichen). Die Kopula sind hat also drei Dependenten: das Subjekt es, das Prädikativ Menschen und den Punkt. Von Menschen hängen wiederum die Kernelemente die und einfachen ab, sowie das Komma und das Wort leiden unter der Relation Relativsatz. Von leiden hängt nur das Subjekt die ab. Wie üblich wird ein künstliches Wurzelwort „root“ angesetzt. Zusätzlich zu der hier dargestellten Information kann auch auf die Wortart und morphologische Information zugegriffen werden.

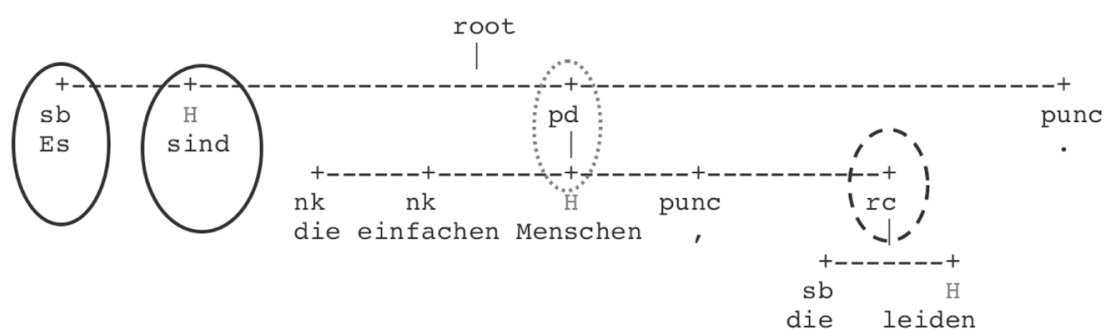

Abb. 1: Dependenzstruktur für (1), mit Markierung der charakteristischen Bestandteile der Spaltsatzkonstruktion

Für die automatische Analyse des Niederländischen wurde der manuell entwickelte Alpino-Parser (van Noord 2006) verwendet. Die Parser für das Deutsche, Englische und Schwedische sind statistische Parser, die wir auf handannotierten Korpora (so genannten Baumbanken) trainierten (der in Bouma et al. 2010 verwendete Parser war der so genannte Malt-Parser, Nivre et al. 2006a). Mit ähnlichen Trainingsverfahren lassen sich mittlerweile Texte aus Zeitungskorpora mit einer Genauigkeit von über 90\% parsen (Bohnet 2010; Seeker et al. 2010a), d.h. neun von zehn Dependenzkanten sind richtig eingetragen und tragen die korrekte Relationsbezeichnung. ${ }^{16}$

Die Ovale in Abbildung 1 deuten die Teile der syntaktischen Konfiguration an, die für die Spaltsatzkonstruktion charakteristisch sind: Die Kopula verbindet sich mit dem Expletivpronomen es als Subjekt, und vom Prädika-

15 Die Entwicklung der Suchfunktionalität und der Darstellung geht vollständig auf Gerlof Bouma zurück.

16 In den Abbildungen hier sind reale Ausgabebeispiele für eine aktuelle Version der Parser aus Bohnet (2010) aufgeführt, ohne Korrektur von evtl. enthaltenen Analysefehlern. (Allerdings wurden die Beispiele nicht zufällig ausgewählt, so dass grobe Anbindungsfehler sicherlich unterrepräsentiert sind.) Beispielsweise kann man in Abbildung 6 im dass-Satz zwei Subjekte sehen (ein typisches Problem bei der Kopula-Konstruktion). 
tiv hängt ein Relativsatz ab (bzw. ist im Fall der Variante, die durch (2) illustriert wird, ein Komplementsatz vorhanden). Ohne hier auf den exakten Formalismus für die Spezifikation eingehen zu wollen, ${ }^{17}$ wird anhand des Beispiels deutlich, dass über die hierarchische Gliederung des Dependenzbaums ein direkter Zugriff auf die strukturell relevanten Bestandteile besteht, unabhängig von ihrer linearen Anordnung in einer konkreten Instanz: Die Baumkonfiguration der Dependenzrepräsentation bleibt bei Wortstellungsvarianten stets gleich. So lässt sich sogar sprachübergreifend ein kompakter Suchausdruck formulieren, der einerseits umfassender als ein regulärer Ausdruck ist (da er Stellungsvarianten automatisch mit berücksichtigt und damit viele falsch negative Befunde vermeidet) ${ }^{18}$ der andererseits aufgrund der direkten syntaktischen Beschränkung der Kandidaten aber auch unerwünschte falsch positive Befunde selbst bereits stärker ausschließt.

Abbildung 2 illustriert, wie beispielsweise beide Spaltsatzinstanzen in dem problematischen Koordinationsbeispiel (7) identifiziert werden (das Expletivpronomen und die Kopula kann „doppelt“ verwendet werden).

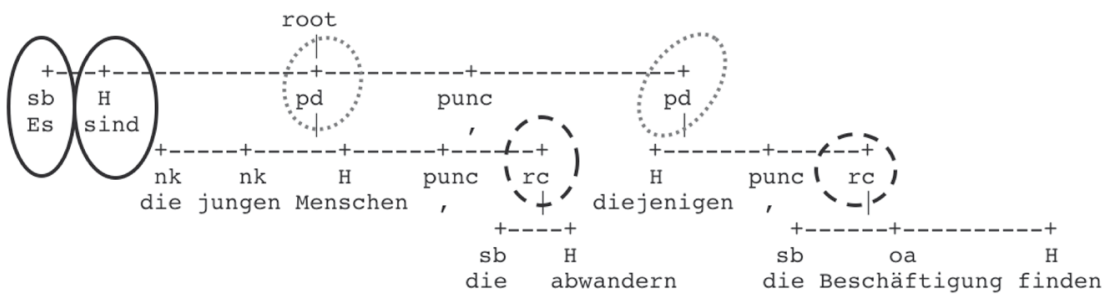

Abb. 2: Analyse für Satz (7)

Natürlich verbleiben auch bei einer syntaktischen Suche unter den vorhergesagten Kandidatenausdrücken falsch positive Befunde, in diesem Fall beispielsweise Sätze mit einem referenziellen $e s$ und einer „echten“ Prädikation mit abhängigem Relativsatz, wie in Satz (8c) im Kontext der Sätze (8a/b).

(8) a. Abschließend möchte ich nochmals betonen, daß wir große Anstrengungen unternehmen müssen, um die Themen dieser Konferenz in der breiten Öffentlichkeit bekannt zu machen.

b. Es sind schwierige Themen.

c. [Es] [sind] [politische und die Institutionen betreffende Themen], [die nicht immer einfach zu erklären sind].

17 In Bouma et al. (2010) wird eine Formulierung der Strukturbeschränkungen durch vordefinierte Templates in der Logik-Programmiersprache Prolog durchgeführt, die sich nach einer Einarbeitungszeit sehr gut handhaben lassen.

18 Man muss sich jedoch bewusst sein, dass aufgrund einer nicht perfekten Parsingqualität Korpusbelege mit der erwarteten Konfiguration übersehen werden können und so falsch negative Befunde entstehen, die hinterher schwer aufzufinden sind. 
Strukturell sind derartige Beispiele nicht von Spaltsätzen unterscheidbar, und damit ist eine automatische Unterscheidung nahezu unmöglich, da sie im allgemeinen Fall eine umfassende semantische Einordnung auf Basis von subtilen Wissensentscheidungen voraussetzen würde. ${ }^{19}$ Für eine sorgfältige Analyse bleibt also eine manuelle Sichtung notwendig. Erfreulicherweise reduziert sich die Zahl der zu sichtenden Belege durch eine systematische syntaktische Suche allerdings erheblich gegenüber regulären Ausdrücken über die Oberflächenfolge.

Ein zweiter Aspekt, der einem beim derartigen Einsatz von Parsern bewusst sein muss, ist, dass ein automatischer Parser nie vollständig korrekte Analysen produzieren wird. Man muss also für jede Anwendung überprüfen, ob das Suchergebnis nicht allzu sehr durch Parsingfehler beeinträchtigt wird. Dabei muss einem bewusst sein, dass nur falsch positive Befunde in der Ergebnisliste direkt beobachtet werden können; um falsch negative Befunde aufzuspüren, ist ein unabhängiger Zugang zu einer Testmenge von korrekten Zieldaten (einem so genannten „Gold-Standard“) erforderlich, beispielsweise durch ein vorab hand-annotiertes Testkorpus (vgl. Fußnote 9).

\subsubsection{Vergleichende Evaluation}

In der modernen Computerlinguistik wird großes Gewicht auf eine systematische Evaluation von vorgeschlagenen Methoden auf unabhängigen empirischen Daten gelegt. In der Tat kann gerade bei automatischen Verfahren der Augenschein beim ersten unsystematischen Sichten von Ergebnissen sehr täuschen. Die Etablierung von automatischen Methoden in der Grammatikforschung sollte also nach Möglichkeit mit einem entsprechenden Kontrollmechanismus einhergehen. Deshalb soll hier auch eine (wenn auch eine auf eine Sprache beschränkte) unabhängige Evaluation der Spaltsatzexperimente wiedergegeben werden.

Zur Evaluation der Vorhersagequalität konnten wir in Bouma et al. (2010) für das Schwedische auf eine Baumbank mit einer expliziten, manuell vorgenommenen Markierung von Spaltsätzen zurückgreifen (Talbanken05, Nivre et al. 2006b). Wir verglichen für unterschiedliche Methoden einer automatischen Bestimmung von Spaltsatzkandidaten, inwieweit diese mit den tatsächlichen (Gold-Standard-)Spaltsätzen nach der manuellen Annotation über-

$19 \quad$ Eine gewisse Verbesserung der automatischen Erkennung durch Ausnutzung von Information außerhalb des Zielsatzes ist damit dennoch nicht ausgeschlossen. Für (8c) könnte beispielsweise der Parallelismus zu Satz (8b) ausgenutzt werden, der keinen Spaltsatz enthalten kann. Alternativ könnte die Übersetzung in eine andere Sprache hilfreich sein; in diesem Fall enthält die englische Version das eindeutig referenzielle Pronomen these:

(i) a. Finally, let me repeat, we shall have a very great effort to make in order to popularise the topics of these negotiations.

b. These are difficult subjects,

c. subjects relating to institutional policy and mechanisms which are not always easy to explain. 
einstimmen. Die Ergebnisse sind in der Tabelle in Abbildung 3 dargestellt. Ich werde auf die Interpretation der wiedergegebenen Zahlenwerte im Folgenden ausführlich eingehen.

\begin{tabular}{|l|c|c|c|}
\hline & \multicolumn{3}{|c|}{ Vorhersagequalität } \\
\hline Methode & Precision & Recall & F-Score \\
\hline Regulärer Ausdruck (eng) & $21,9 \%$ & $47,8 \%$ & $30,1 \%$ \\
\hline Regulärer Ausdruck (breit) & $11,1 \%$ & $88,6 \%$ & $19,7 \%$ \\
\hline $\begin{array}{l}\text { Syntaktischer Ausdruck } \\
\text { (mit perfektem Parser) }\end{array}$ & $53,0 \%$ & $84,1 \%$ & $65,0 \%$ \\
\hline $\begin{array}{l}\text { Syntaktischer Ausdruck } \\
\text { (mit automatischem Parser) }\end{array}$ & $43,8 \%$ & $54,7 \%$ & $48,7 \%$ \\
\hline
\end{tabular}

Abb. 3: Ergebnis eines Vergleichs von verschiedenen automatischen Verfahren für die Spaltsatzsuche in dem schwedischen Talbanken05-Korpus

Als Precision wird bei derartigen Evaluationen der Prozentsatz der korrekt vorhergesagten Instanzen unter allen vorhergesagten Kandidaten angegeben; als Recall der Anteil der tatsächlich im Korpus vorhandenen Spaltsätze, die auch gefunden wurden. Der F-Score ist ein Mittelwert, der zeigt, ob nicht eines der beiden Kriterien zu Ungunsten des anderen optimiert wurde (beispielsweise könnte man 100\% Recall erreichen, indem man schlichtweg jeden Satz im Korpus als Kandidaten ausgibt, die Precision wäre dann jedoch sehr bescheiden). In der Regel wird ein möglichst hoher F-Score-Wert angestrebt - je nach Anwendungskontext des Verfahrens kann jedoch auch stärkeres Gewicht entweder auf Precision oder auf Recall gelegt werden. (Sind beispielsweise Ressourcen für eine aufwändige manuelle Nachanalyse verfügbar, wird z.B. dem Recall mehr Gewicht zu geben sein.)

$\mathrm{Da}$, wie in Abschnitt 3.1 erläutert, bei regulären Ausdrücken ein Kompromiss zwischen engen und umfassenderen Formulierungen der Suchanfrage gefunden werden muss, wurden zwei Ausdrücke mit unterschiedlicher Schwerpunktsetzung getestet. Das Schwedische hat ebenso wie das Deutsche eine relativ freie Wortstellung. Beim „engen“ Ausdruck wurde nur die kanonische Spaltsatzreihenfolge wie in (3) angesetzt, in der „,breiten“ Variante wurden Stellungsvarianten explizit aufgenommen (und die Möglichkeit, im Schwedischen Relativsätze ohne die subordinierende Konjunktion som zu verwenden, wurde berücksichtigt). Wie nicht anders zu erwarten, zeigt der breite Suchausdruck mit über 88\% im Vergleich zu knapp 48\% einen wesentlich höheren Recall - allerdings bewegt sich die Precision in beiden Fällen auf einem sehr bescheidenen Niveau: Beim engen Ausdruck ist nur etwa ein Beleg von fünf vorhergesagten ein echter Treffer, beim breiten Ausdruck sogar nur einer von neun. 
Im Verhältnis zu diesen beiden Verfahren ist nun das syntaktische Verfahren zu beurteilen. Zur Abschätzung des Einflusses der Analysequalität des automatischen Parsers ist es üblich, einen Vergleich zwischen einem (realistischen) Einsatz des automatischen Parsers einerseits und dem hypothetischen Einsatz eines perfekten Parsers andererseits zu machen. Auf den Testdaten (im Gegensatz zu späteren Parser-Anwendungen auf neuen Daten) kann man einen perfekten Parser simulieren, indem man einfach die manuell annotierten syntaktischen Analysen verwendet.

Die Evaluationsergebnisse für die Spaltsatzsuche (in den beiden untersten Zeilen in Abbildung 3) zeigen, dass die Precision des syntaktischen Verfahrens sehr deutlich über dem klassischen Einsatz von regulären Ausdrücken liegt - mit knapp 44\% auch unter realistischen Bedingungen mit dem automatischen Parser.

Beim Recall ist der Vorteil des syntaktischen Verfahrens im Falle der automatischen Analyse nicht ganz so deutlich: Da die syntaktische Analyse recht detailliert in den Suchausdruck eingeht, ist es nicht verwunderlich, dass Parsing-Fehler hier deutlich zu Buche schlagen: Erkennt der Parser beispielsweise das Prädikativ nicht korrekt, kann der auf der Analyse aufbauende Suchausdruck die Konfiguration nicht mehr finden. Mit einem perfekten Parser wären rund $84 \%$ Recall möglich, mit dem automatischen Parser noch knapp 55\%. ${ }^{20}$ Damit liegt aber auch der automatische Parser noch über dem Recall des engen regulären Suchausdrucks (mit knapp 48\%), den man zur Vermeidung von nahezu endlosen Kandidatenlisten vermutlich in vielen Experimenten gegenüber dem breiten Ausdruck bevorzugen würde.

Wichtig ist vor allem, dass auch beim automatischen Parser der gemittelte F-Score-Wert mit knapp 49\% sehr deutlich über den F-Score-Werten der regulären Ausdrücke (mit knapp 20\% bzw. mit 30\%) liegt. Dies zeigt, dass die syntaktischen Verfahren im Abwägen zwischen dem möglichen Übersehen eines Belegs und der Vermeidung von allzu langen Kandidatenlisten wesentlich effektiver ist. ${ }^{21}$

20 Eine Optimierung der Parsing-Qualität gegenüber den Experimenten in Bouma et al. (2010) ist denkbar. Darüber hinaus ließe sich die Gesamtqualität des gesamten Suchverfahrens möglicherweise verbessern, wenn die Suchausdrücke nicht direkt auf (als korrekt angenommenen) Dependenzkonfigurationen formuliert würden, sondern wenn auch die Belegsuche mit einem trainierbaren statistischen Verfahren realisiert würde. Es würde also eine zusätzliche Trainingsphase zwischengeschaltet, in der sich das System auf typische Fehler im ersten Parsing-Schritt einstellen könnte.

21 Es mag manchen Leser verwundern, dass ich den breiten regulären Ausdruck mit dem insgesamt höchsten Recall (über 88\%) nicht generell als die beste Wahl für eine sprachwissenschaftliche Studie einstufe - wo es doch bei einer sorgfältigen wissenschaftlichen Untersuchung nicht wie in der Sprachtechnologie um Zeitersparnis oder ähnliche Überlegungen, sondern um größtmögliche Qualität gehen muss. Hierzu ist zweierlei zu sagen: Erstens dürfte es im Zuge einer ausgedehnteren Studie untergeordnete Teilfragen geben (evtl. z.B. zu einem Vergleichsphänomen oder einer Kontrastsprache), für die der Blick auf die Datenlage überhaupt nur mit der Möglichkeit einer zügigen Datenexploration realistisch wird. Solange eine 


\subsubsection{Diskussion der auf automatischen Analysen basierenden Struktursuche}

Die ausführliche Wiedergabe und Erläuterung des Vergleichsexperiments aus Bouma et al. (2010) an dieser Stelle kann sicherlich nicht mehr leisten als die Illustration eines - hoffentlich jedoch typischen - Fallbeispiels für eine computerlinguistisch informierte Struktursuche. Es dürfte jedoch deutlich geworden sein, dass bei einem derartig gelagerten Fall die Verfügbarkeit von „tieferen" linguistischen Annotationen die Korpussuche erheblich vereinfachen kann, selbst dann, wenn wegen der großen Datenmengen nur mit einer automatischen Analyse gearbeitet werden kann. Gerade für den Sprachvergleich kann eine syntaktische Suchkonfiguration sehr einfach auf eine Vergleichssprache angewandt werden. (Wie die Erfahrung zeigt, ist für eine explorative Untersuchung nicht einmal eine große Detailkenntnis zu allen Aspekten der Grammatik der Sprache notwendig, sofern das Beschreibungsinventar der Annotationen sinnvoll gewählt wurde.)

geeignete Fehlerabschätzung gewährleistet, dass die Ergebnisse nicht überinterpretiert werden, können diese explorativen Studien möglicherweise entscheidende Erkenntnisse für die Bearbeitung der eigentlichen Kernfrage liefern (die dann mit der größtmöglichen Sorgfalt durchgeführt wird).

Zweitens könnte man sich bei einer auf absolute Vollständigkeit ausgerichteten Fragestellung auch mit den über $88 \%$ Recall des breiten regulären Ausdrucks nicht zufrieden geben. $100 \%$ Recall sind, wie oben bereits gesagt, immer zu erreichen, indem schlichtweg jeder Satz im Korpus als ein Kandidat betrachtet wird. Die manuelle Sichtung müsste also ungefiltert über das Gesamtkorpus gehen. Könnte man sich aber dann sicher sein, dass bei einem seltenen Phänomen nicht Ermüdungserscheinungen dazu führen, dass es zu Fehleinschätzungen kommt?

An diesem Extrembeispiel dürfte deutlich werden, dass die Precision eines Verfahrens unter realistischen Bedingungen auch bei einer anschließenden manuellen Sichtung eine Rolle spielt. Dazu noch eine Beispielrechnung: Bei einem Korpus von 10.000 Sätzen, das in der Verteilung den schwedischen Testdaten entspricht (ca. $2 \%$ der Sätze enthalten einen Spaltsatz), würde der breite reguläre Ausdruck eine Liste von etwa 1.600 Kandidaten zur manuellen Nachsichtung liefern, von denen 177 korrekt wären. Setzt man pro Satz (willkürlich) eine mittlere Bearbeitungszeit von einer halben Minute an, würde die Sichtung der Ergebnisliste über 13 Stunden in Anspruch nehmen. Wenn man als Gedankenspiel außerdem ansetzt, dass bei der Sichtung gleichmäßig verteilt in einem von 30 Fällen eine Fehlentscheidung erfolgt, so würden sich wegen der langen Liste so viele Fehler einschleichen, dass die Precision der komplett handgefilterten Daten nur gut 76\% beträgt (Recall $85,7 \%$ ). Dagegen wäre mit dem syntaktischen Verfahren - sofern die 10.000 Sätze aus einer Baumbank stammten, wenn also perfekte syntaktische Analysen zur Verfügung stünden - nur eine Liste von knapp 320 Kandidaten zu sichten (Zeitbedarf knapp über 2 $\frac{1}{2}$ Stunden); bei gleicher Fehlerquote in der manuellen Sichtung würde sich eine Precision von fast 94\% ergeben (Recall 81\%). Ohne Baumbankinformation, also bei automatischem Parsing wären 250 Kandidaten zu sichten (in rund 2 Stunden), mit einem Ergebnis von 92,7\% Precision (und knapp 53\% Recall). Natürlich lassen sich durch doppelte Kontrolle manuelle Fehler eindämmen - mit entsprechend nochmals erhöhtem Zeitaufwand; in aller Regel wird man bei langen Listen jedoch zusätzliche Filter verwenden, die nie garantiert fehlerfrei sind. Das fiktive Rechenbeispiel illustriert also recht klar: Wenn für den ursprünglichen Suchausdruck eine verhältnismäßig hohe Precision erreicht werden kann, ist dies fast immer von Vorteil. 
Die Spaltsatzkonstruktion mag ein verhältnismäßig günstiges Beispiel sein, da die zugehörige Dependenz-Konfiguration aus für sich jeweils relativ häufigen „Komponenten“ besteht und sich so verhältnismäßig robust erkennen lässt. Es ist nicht auszuschließen, dass andere Objekte des sprachvergleichenden Interesses eine größere Schwierigkeit darstellen. Wenn beispielsweise eine sehr seltene syntaktische Konstruktion X leicht mit einer häufigen Konstruktion Y verwechselt werden kann, ist es wahrscheinlich, dass beim automatischen Parsen fast ausschließlich die Analyse für Y erzeugt wird, so dass der Vorteil einer tieferen Analyse bei der Suche nach X-Instanzen möglicherweise reduziert wird.

Die generelle Tragfähigkeit des Ansatzes müssen weitere praktische Studien zeigen. Die Grenzen des geschilderten Verfahrens stellen jedoch keine grundsätzlichen Grenzen dessen dar, was mit kombiniert linguistisch/statistischen Methoden möglich ist. Wenn die Standard-Analysen, die ein gegebener automatischer Parser erzeugt, in einem speziellen Fall ungeeignet sind, schließt das nicht aus, dass eine automatische Identifizierung der gewünschten Zielkategorie aus der verfügbaren Korpusinformation möglich ist. Um die weitergehenden Möglichkeiten auszuloten, ist dann allerdings eine stärkere Verwobenheit der Grammatikforschung und der computerlinguistischen Forschung erforderlich, wie ich sie in der Einleitung angedeutet habe und unten weiter ausführen werde.

\subsection{Parallelkorpora und Sprachvergleich}

Bislang standen in diesem Aufsatz die Möglichkeiten einer automatischen Strukturanalyse im Vordergrund, mit der wesentlich größere Mengen von Korpusdaten für die Grammatikforschung zugänglich gemacht werden können, als sie im Rahmen von vorab annotierten Baumbanken zur Verfügung stehen. Vorteile für einen spezifisch sprachvergleichenden Einsatz dieser Methode erscheinen dabei zunächst nicht allzu offensichtlich - obwohl es auch sie gibt: Während die Grammatikforscherin oder der Grammatikforscher bei der Arbeit mit existierenden manuell annotierten Baumbanken auf die für die jeweiligen Sprachen vorhandene Korpusauswahl und das Annotationsformat eingeschränkt ist, können bei der Arbeit mit Parsern unter Umständen wesentlich besser vergleichbare Korpora zugrunde gelegt und dann automatisch nach sprachübergreifend gleichartigen Kriterien analysiert werden. Je nach verwendetem Parsing-Ansatz können möglicherweise sogar Inkonsistenzen im Annotationsformat ausgeglichen werden: Beim Training eines deutschen Parsers auf einer Dependenz-Variante der TIGERBaumbank kann beispielsweise die bisweilen irritierende interne Struktur von Präpositionalphrasen systematisch korrigiert werden (Seeker/Kuhn 2011). 
Darüber hinaus bietet es sich jedoch an, in der sprachvergleichenden Forschung auch direkt mit Parallelkorpora zu arbeiten. ${ }^{22}$ Hier kann von anderen jüngeren Entwicklungen in der maschinellen Sprachverarbeitung profitiert werden, was im Folgenden kurz illustriert werden soll. Hauptsächlich durch die Forschung zu statistischen Verfahren für die maschinelle Übersetzung wurden vielfältige vollautomatische Analysetechniken für Parallelkorpora entwickelt.

Für jede Arbeit mit Parallelkorpora ist zunächst eine Bestimmung von Satzentsprechungen unerlässlich (und bei einer Größenordnung von Millionen von Sätzen muss dieser Schritt vollautomatisch durchführbar sein eine sprachunabhängige Nummerierung der übersetzten Einheiten ist nur in den seltensten Fällen bereits im Roh-Korpus enthalten, die Buch-/Kapitel-/ Versnummerierung in der Bibel ist ein solches Beispiel). Wenn die Satzentsprechung im Roh-Korpus nicht allzu stark von einer parallelen linearen Folge abweicht (wenn es bei der Übersetzung also nicht zu größeren Löschungen, Ergänzungen oder Umstellungen von ganzen Textabschnitten kam), lässt sich dieser Schritt nach Gale/Church (1993) überraschend gut vollautomatisch anhand des Verhältnisses der Satzlängen vornehmen - wobei selbstverständlich ein Satz in der einen Sprache mehreren Sätzen in der anderen entsprechen kann (vgl. Beispiel (8) und seine englische Entsprechung in der Fußnote).

Je nach Aufgabenstellung können schon die automatisch berechneten Satzentsprechungen auf großen Parallelkorpora eine sehr große Hilfe für die vergleichende Forschung sein. Für stärker lexikalisch orientierte Fragestellungen können über die Internet-Anfrage ${ }^{23}$ zum OPUS-Korpus (http:// opus.lingfil.uu.se/) beispielsweise schnell alle sprachübergreifenden Satzentsprechungen gefunden werden, die bestimmten Filtern zur Wort- oder Wortartenkombination (in einer oder mehreren beteiligten Sprachen) genügen.

Rein statistische Verfahren zur Erschließung von wahrscheinlichen Wortentsprechungen eröffnen jedoch prinzipiell auch einen feingliedrigeren Zugang für den systematischen Sprachvergleich unterhalb der Satzebene. Die entscheidenden Arbeiten hierzu wurden Anfang der 1990er Jahre am IBM T.J. Watson Research Center durchgeführt (Brown et al. 1990, 1993). Mit mathematisch sehr anspruchsvollen Verfahren und unter Ausnutzung der in den letzten Jahren stark angewachsenen verfügbaren Rechenkapazität werden die Wort-Okkurrenzen in zwei Vergleichssprachen in einem mög-

22 Dabei ist es nicht selbstverständlich, dass Parallelkorpora - so sie vorhanden sind - gegenüber anderen Datenquellen zu bevorzugen sind: Das Nebeneinander von quellsprachlichen Originaltexten und Übersetzungsprodukten muss (je nach Untersuchungsgegenstand) sorgfältig beachtet werden. Mitunter mag es besser sein, „nur“" vergleichbare Korpora zu verwenden, die jedoch ausschließlich aus Originaltexten bestehen.

23 Die Anfrageschnittstelle beruht auf der IMS Corpus Workbench (Christ 1994). 
lichst großen Parallelkorpus zueinander in Beziehung gesetzt. Das Ergebnis eines längeren Prozesses der zyklischen Verfeinerung ist (für die Zwecke der sprachvergleichenden Forschung) eine so genannte statistische Wortalignierung - d.h. für jeden Satz im Parallelkorpus kann für ein Wort in Sprache 1 angegeben werden, welche Wörter in Sprache 2 die wahrscheinlichsten Entsprechungen sind. In Abbildung 4 ist eine derartige Wortalignierung für die Sprachpaare Schwedisch/Deutsch und Deutsch/Englisch durch die Verbindungslinien angedeutet.

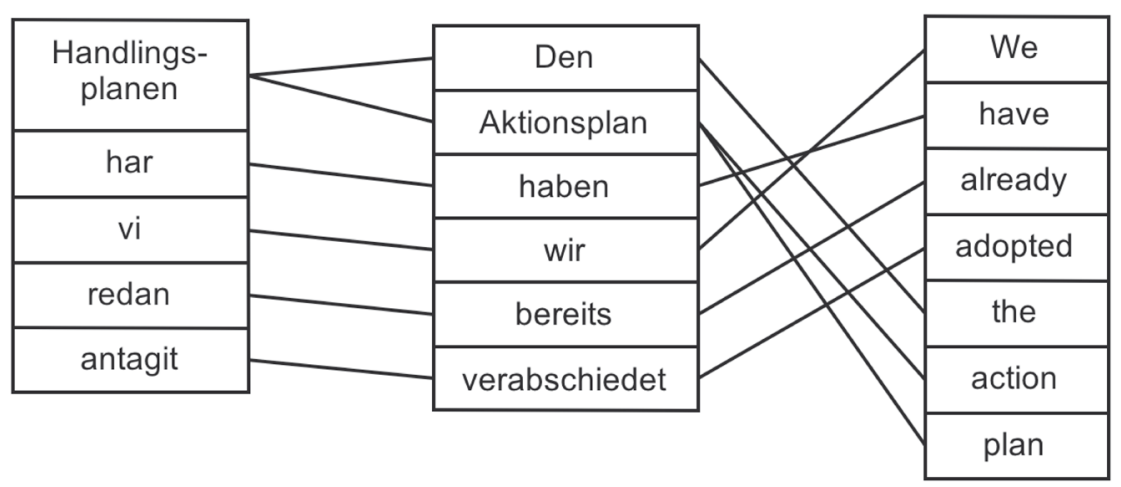

Abb. 4: Wortalignierung für ein schwedisch-deutsch-englisches Beispiel

Das Verfahren der statistischen Wortalignierung folgt bei aller Komplexität einem sehr einfachen Gedanken: ein Wort $w$ aus Sprache 1, das im gesamten Parallelkorpus vor allem dann auftritt, wenn der entsprechende Satz in Sprache 2 ein Wort $w^{\prime}$ enthält, ist mit großer Wahrscheinlichkeit (ein Teil von) dessen Übersetzungsentsprechung. Beispielsweise werden viele der deutschen Sätze, die das Adverb bereits enthalten, in ihrer englischen Entsprechung das Adverb already enthalten. Diese Beobachtung lässt sich ohne jedes Wissen über die Sprachen ausnutzen. Selbstverständlich sind die Verhältnisse komplizierter: Viele Wörter sind mehrdeutig und werden von Fall zu Fall unterschiedlich übersetzt; Übersetzungseinheiten bestehen aus mehreren Wörtern; strukturelle Unterschiede oder stilistische Überlegungen führen zu Abweichungen von einer wörtlichen Übersetzung; oft lässt sich einzelnen Wörtern gar keine direkte Entsprechung zuordnen. Das zyklische Verfahren ist jedoch in der Lage, die „einfachen“ Fälle in einer Art Ausschlussverfahren als Grundlage für die Berechnung von wahrscheinlichen Entsprechungen auch in den ,komplizierten“ Fällen zu nutzen und erreicht eine überraschend gute Qualität in der endgültigen Wortalignierung.

In der sprachtechnologisch orientierten Forschung gibt es vielfältige Anwendungen für eine so bestimmte Wortalignierung, insbesondere die Idee der „Annotationsprojektion“, die grundsätzlich auf Yarowsky et al. (2001) zurückgeht: Wenn für eine der Sprachen im Parallelkorpus (häufig das Eng- 
lische) verhältnismäßig zuverlässige Annotationen für eine grobkörnige grammatische Kategorisierung verfügbar sind (beispielsweise durch eine qualitativ hochwertige automatische Bestimmung der Wortarten), kann die Wortalignierung genutzt werden, um diese Kategorisierung als Approximation auf eine andere Sprache im Parallelkorpus zu übertragen (beispielsweise eine computerlinguistisch wenig untersuchte Sprache wie die senegalische Sprache Wolof in Dione et al. 2010). Trotz bekannter Abweichungen von einer sprachübergreifenden Entsprechung der grammatischen Kategorien kann das massive statistische Überwiegen der kanonischen Fälle im Ergebnis zu einem befriedigenden Resultat führen - vor allem, wenn robuste Lernverfahren eingesetzt werden, die in der Lage sind, Abweichungen vom kanonischen Fall aufgrund des Kontextmusters zu korrigieren. So lassen sich für so genannte „ressourcenarme“ Sprachen ohne großen Aufwand immerhin Basis-Analysewerkzeuge erzeugen.

Ob sich ein derartiges Projektionsverfahren für vollautomatische Werkzeuge in der sprachvergleichenden Grammatikforschung mit Gewinn einsetzen lässt, ist sicherlich fraglich - liegt doch das Augenmerk gerade auf den Fällen der Divergenz im kontrastiven Vergleich. In Kombination mit einer robusten und zuverlässigen automatischen syntaktischen Analyse für die Einzelsprachen ergibt sich jedoch aus der statistischen Wortalignierung - nach meinem Dafürhalten - ein erhebliches Potenzial für die empirische Forschung, das noch längst nicht voll erkundet wurde.

Dies sei anhand von einigen Beispielen zur Thematik der Spaltsatzkonstruktion illustriert. Hier kann (und analog ist das für andere syntaktische Konstruktionen denkbar) jeweils ein Suchausdruck für die grammatische Konfiguration in einer oder mehreren Einzelsprachen verwendet werden (wie in Abschnitt 3.2 diskutiert), deren Treffer dann über die statistische Wortalignierung zueinander in Beziehung gesetzt werden können, so dass ein kontrastiver Vergleich nicht nur auf der Wortebene, sondern auch auf einer strukturellen Ebene unterstützt werden kann. Für das deutsch-englische Übersetzungspaar (9) ist dies in Abbildung 5 illustriert. Die beiden Dependenzbäume für die deutsche und englische Satzanalyse ${ }^{24}$ folgen den

24 Einige Bemerkungen zur automatischen Analyse der deutschen Satzstruktur für (9a): Die Nominalphrase genau dieses Verfabren in Abbildung 5 wird beim automatischen Parsen überraschenderweise als Dativobjekt („da“) analysiert. Kennt man das Vorgehen des robusten Parsers, erscheint diese Fehlentscheidung allerdings nicht gar so absurd: Um jede der vielen Teilentscheidungen beim Parsen aus einer jeweils möglichst großen Anzahl von Trainingsdaten ableiten zu können, wird der „Blick“ des Parsers nur auf einige charakteristische Teilrepräsentationen gelenkt. Um zu entscheiden, ob Verfahren ein Dependent von baut ist, und wenn ja, unter welcher Relation, steht zunächst nur die morphologische Form der beiden Wortknoten bereit (und die enthält bei Verfabren einen Kasussynkretismus; außerdem dürfte bauen in vielen Verwendungen im Korpus mit einem Dativ aufgetreten sein). Um zusätzliche Information aus der Nominalphrase auszunutzen, wird außerdem der am weitesten links gelegene Dependent von Verfahren berücksichtigt. In den allermeisten Fällen wird dies der 
Konventionen aus Abschnitt 3.2, wobei der englische Baum mit der Wurzel nach unten dargestellt ist, so dass die statistische Wortalignierung direkt zwischen den Bäumen eingetragen werden kann.

a. Genau dieses Verfahren baut jedes Jahr wieder Vertrauen ab.

b. [It] [is] [precisely this process] [that undermines confidence every year].

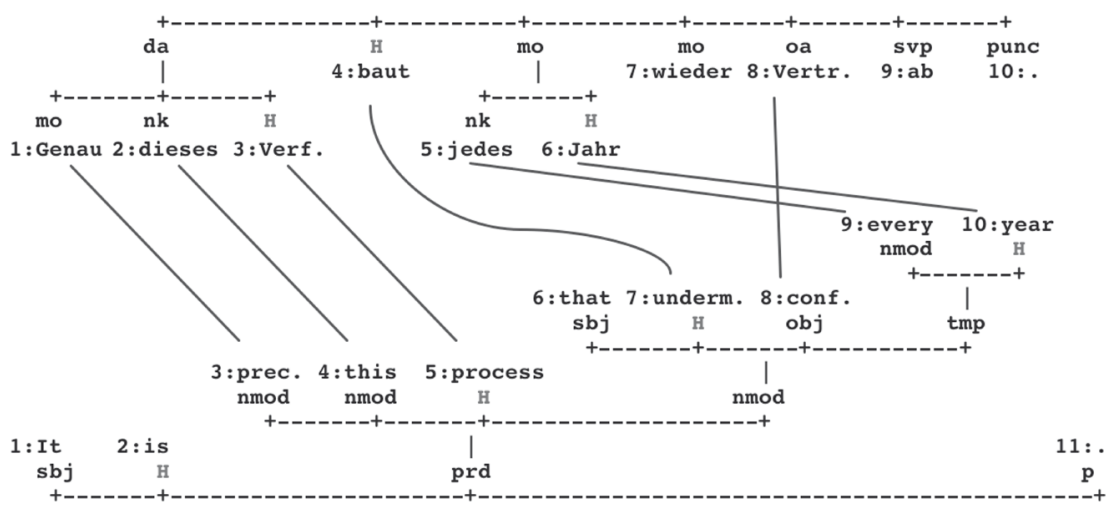

Abb. 5: Die parallelen, mit einer statistischen Wortalignierung verbundenen automatischen Satzanalysen für das Übersetzungspaar $(9 \mathrm{a} / \mathrm{b})$

Die gezeigte Alignierung entspricht der tatsächlichen Systemausgabe und zeigt, dass sich in diesem Fall die Entsprechung der relevanten Bereiche der Spaltsatzkonstruktion ohne weiteres automatisch abgleichen ließe. In Abbildung 6 ist die automatische Analyse und Alignierung für das Übersetzungspaar (10) dargestellt, in dem der deutsche Spaltsatz im Englischen einem so genannten reversed wh-cleft bzw. pseudo cleft (Pseudospaltsatz bzw. Sperrsatz) entspricht.

Artikel sein, der die Kasusentscheidung stark eingrenzt - hier ist es jedoch die wenig hilfreiche Fokuspartikel genau. Das eigentlich entscheidende Demonstrativpronomen fällt in diesem Fall aufgrund allgemeiner Beschränkungen unter den Tisch.

Was vielleicht überraschen mag, ist, dass keine übergeordneten Valenzbeschränkungen den subjektlosen Satz ausschließen. Es erweist sich jedoch als sehr schwierig, solche globalen Kriterien in robuster Weise einzubringen. Da die meisten Verben in realen Korpusdaten sehr variable Valenzmuster aufweisen, besteht die Gefahr, dass die globalen Beschränkungen zu mehr ungerechtfertigten Korrekturen führen als sie echte lokale Fehler korrekt identifizieren.

In unserem laufenden Projekt zum datengesteuerten Dependenz-Parsing (Teilprojekt D8 im SFB 732 ,Inkrementelle Spezifikation im Kontext') arbeiten wir jedoch an einem systematischen Verfahren für eine „konservative“ Berücksichtigung von globalen linguistischen Beschränkungen (auf der Basis der Vorarbeiten in Seeker et al. 2010b). Beispielsweise lassen sich so die Kongruenzbedingungen in der Nominalphrase genau dieses Verfabren durch eine unterspezifizierte Repräsentation sehr zuverlässig erfassen. 
(10) a. Aber noch wichtiger ist, daß [es] [die kulturellen Aktivitäten sind], [die der Menschheit Zivilisation verleihen].

b. But, more importantly than that, cultural activities are what make mankind civilised.

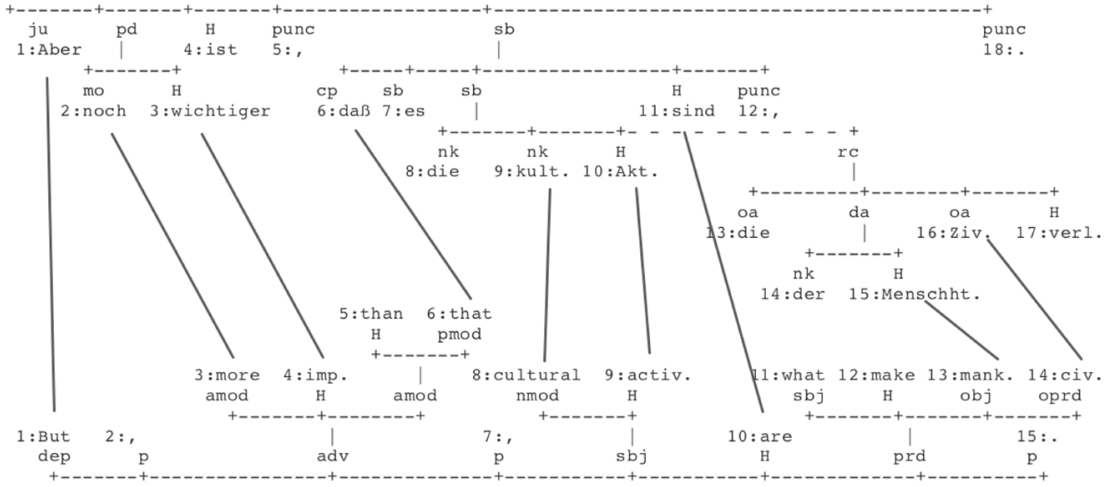

Abb. 6: Automatische Analyse und Wortalignierung für das Übersetzungspaar (10a/b) (mit fehlerhafter Alignierung von daß und that)

Abbildung 6 enthält einen klaren Alignierungsfehler, der sich leicht aus den Mechanismen der statistischen Alignierung erklären lässt: Die subordinierende Konjunktion daß im deutschen Satz ist fälschlich mit dem englischen that aligniert, das in diesem Fall jedoch ein Demonstrativpronomen ist. Durch die unterschiedlichen Konstruktionen, wie sie im Übersetzungsprozess angeführt wurden, gibt es weder für daß noch für that in der jeweils anderen Sprache eine tatsächliche wörtliche Entsprechung. Die Alignierung, die auf Basis der Wortformen vorgeht, folgt jedoch der naheliegenden Tendenz, die beiden Wortformen zu alignieren, zumal beide zufällig in der Wortfolge an entsprechender Stelle (jeweils als 6. Wortform) auftauchen.

Selbst mit dem Alignierungsfehler dürfte die automatische Analyse jedoch für einen Abgleich der Konstruktionsteile bei einer Spaltsatzkonstruktion im Deutschen mit einem Englischen pseudo cleft verwendbar sein.

Für eine umfassende Einschätzung der Brauchbarkeit müssten detaillierte Experimente im größeren Maßstab durchgeführt werden. Die prinzipielle Verwertbarkeit geht jedoch bereits aus einer erfolgreichen explorativen Suche hervor, die zur Abrundung dieses Abschnitts anhand einer Reihe von Parallelbelegen illustriert werden soll. Es handelt sich hier um diverse deutsche Entsprechungen zu einer im Englischen beobachteten Spaltsatzkonstruktion - im weiteren Verlauf aus Platzgründen ohne explizite Aufführung der Wortalignierung, jedoch mit kursiver Hervorhebung (der Entsprechung) des „Fokus“ der Spaltsatzkonstruktion und mit Unterstreichung (der Entsprechung) des Relativsatzes. An dieser Stelle sei erneut darauf hin- 
gewiesen, dass ich hier keinen Versuch einer ernsthaften und linguistisch fundierten vergleichenden Untersuchung machen will (die zudem in den Kontext einer reichen vorhandenen Literatur zu diesem Thema zu setzen wäre, wofür hier ebenso wenig der Platz ist, vgl. aber Gast/Wiechmann in diesem Band). Es handelt sich lediglich um einige anekdotische Beispiele.

Die Beispiele (11)-(13) illustrieren, dass es im Deutschen zur Markierung der Informationsstruktur häufig genügt, von der freien Wahl bei der Vorfeldbesetzung Gebrauch zu machen. Dies scheint ausreichend zu sein, um der Konstituente Emphase (bzw. Abtrennung vom Restsatz) zu verleihen, für die das Englische die Spaltsatzkonstruktion benötigt. ${ }^{25}$ Dies ist, wie in (13) zu sehen, sogar bei einem pronominalen Subjekt möglich (da das Subjekt im Deutschen ja bei der Vorfeldbesetzung stets in Konkurrenz mit anderen Konstituenten steht) - in Verbindung mit der Partikel nämlich. Dies erklärt sicherlich zum Teil, warum im Deutschen weniger Spaltsätze als im Englischen verwendet werden, insbesondere weniger Spaltsätze mit einem hervorgehobenen Pronomen. ${ }^{26}$

(11) a. ... ihnen verwehrt man die Zulassung zu einem Praktikum ...

b. [It] [is] [they] [who are refused access to training courses] ...

(12) a. Damit wird eine Lösung möglich.

b. [It] [is] [this] [which will enable a solution to be found].

a. Wir sind nämlich hier die Haushaltsbehörde ...

b. [It] [is] [we] [who are the budgetary authority] ...

Ein seltener Fall eines (stellungsmäßig umgekehrten) deutschen Spaltsatzes mit einem hervorgehobenen Pronomen findet sich in (14a) - der pronominale Bezug von sie bzw. they im Kontext ist die srilankische Regierung). Das Original ist in diesem Fall die englische Äußerung.

a. Aber [sie] [ist] [es] doch, [die kontrolliert, was wir wissen], [sie] [ist] [es], [die kontrolliert, welche Meldungen uns erreichen].

b. Yet [it] [is] [they] [who are controlling what we know] and [it] [is] [they] [who control what coverage we get] ...

25 Bei (12) und (13) war Deutsch die Originalsprache. (11a) und (11b) sind beides Übersetzungen aus dem Französischen; der wiedergegebene Satz ist hier Teil einer längeren Folge von parallelen Sätzen, die im Französischen jeweils durch einen Spaltsatz realisiert ist (der Bezug ist eine Gruppe von Jugendlichen - ces jeunes):

(i) a. C'est à leurs petits frères et leurs petites sœurs qu'on refuse une inscription dans les établissements scolaires,

b. c'est à eux que l'on refuse l'accès aux stages,

c. c'est à leur famille que l'on refuse l'accès à un logement, aux soins de santé.

26 Eine entsprechende Beobachtung macht Johansson (2001) für den Vergleich zwischen dem Englischen und Schwedischen: Im Schwedischen finden sich in Spaltsätzen noch mehr hervorgehobene anaphorische Elemente als im Englischen. 
Als abschließende Beispiele für mögliche Ergebnisse aus automatischer Analyse und Alignierung, die sich selbst bei einer gewissen Fehlerquote recht zuverlässig ermitteln lassen, zeigen (15) und (16) zwei Fälle von Kreuzkonfigurationen in der Übersetzungsentsprechung bei Spaltsätzen im Englischen: In (15) wird im Deutschen die wenn-dann-Konstruktion verwendet, in der die Entsprechung zum Spaltsatz-,,Fokus“ naturgemäß am Ende steht. ${ }^{27}$ In (16) zeigt sich, dass durch die Möglichkeit der VP-Topikalisierung im Deutschen eine Trennung der informationsstrukturellen Bereiche auch ohne Spaltsatzkonstruktion möglich ist. (Die englische Version (16b) ist die Originalfassung.)

(15) a. Wenn jemand eine Lösung des Konflikts behindert hat, dann die marokkanische Regierung.

b. [It] [is] [the Moroccan Government] [that has been putting obstacles in the way of resolving the conflict].

(16) a. Am stärksten belastet ist die Theiß, Ungarns zweitgrößter ... Fluß.

b. [It] [is] [the Tisza, Hungary's second river ...] [that has borne the brunt].

Zusammenfassend kann gesagt werden, dass zumindest für spezielle Konstruktionen, für die keine große Verwechslungsgefahr mit wesentlich häufigeren Standard-Konstruktionen besteht, eine automatische syntaktische Satzanalyse in Kombination mit einer rein statistischen Wortalignierung auf großen Parallelkorpora den Blick für die vergleichende Grammatikforschung potenziell sehr schnell und mit geringem manuellen Aufwand auf interessante kontrastive Belege lenken kann. Für eine quantitative Analyse der automatisch ermittelten Belege müsste im Einzelfall eine sorgfältige Abschätzung von möglichen Verzerrungseffekten vorgenommen werden.

\section{Das Verhältnis zwischen Grammatikforschung und Computerlinguistik}

Nach der ausführlichen exemplarischen Illustration und Diskussion der vermuteten Potenziale, die in einem stark erweiterten Werkzeugeinsatz in der empirisch ausgerichteten (vergleichenden) Grammatikforschung stecken, will ich in diesem Abschnitt den Versuch einer Rekonstruktion machen, die den Status Quo eines eher zögerlichen Einsatzes der eigentlich verfügbaren Möglichkeiten erklärt. Auf dieser Basis kann im abschließenden Ausblick (in Abschnitt 5) über Schlussfolgerungen für das zukünftige Vorgehen nachgedacht werden.

$27 \quad$ Im spanischen Original zu (15) findet sich ein Pseudospaltsatz:

(i) $[\ldots]$ es decir, que quien ha estado poniendo obstáculos a la resolución del conflicto ha sido el Gobierno de Marruecos. 
4.1 Grammatikforschung und „klassische“ Computerlinguistik

Die „klassische“ Computerlinguistik versteht sich als Fortsetzung der formalen Sprachwissenschaft, die grammatisches und lexikalisches Wissen so präzise zu charakterisieren versucht, dass Strukturanalysen mit dem Computer durchgeführt werden können - in einer Weise, die den vielfältigen ebenenübergreifenden Interaktionen und Mehrdeutigkeiten gerecht wird. Der klassische Ansatz dominierte die computerlinguistische Forschungslandschaft bis in die 1990er Jahre, anschließend trat der statistische Ansatz zu ihm in Konkurrenz und gewann an Einfluss, ohne ihn jedoch gänzlich zu verdrängen.

Die formal präzise Umsetzung von linguistischen Erkenntnissen auf dem Computer setzt ein tiefes Verständnis der verwendeten Beschreibungsformalismen voraus, insbesondere zu deren Verarbeitungseigenschaften. Ein möglicher Rückfluss von methodischen Erkenntnissen aus dieser computerlinguistischen Forschungsrichtung in die einzelsprachlichen Sprachwissenschaften oder in die sprachvergleichende Grammatikforschung konzentriert sich jedoch in aller Regel auf die systematische Charakterisierung von bestimmten grammatischen Phänomenen, ist also nicht grundsätzlich verschieden von der Art des Rückflusses von Erkenntnissen aus formal-linguistischen Betrachtungen, die ohne Computereinsatz auskommen. Mit anderen Worten: In dieser Tradition erscheint die Beschäftigung mit spezifisch computerlinguistischen Fragestellungen und Methoden aus Sicht der Grammatikforschung entbehrlich. Die klassische Computerlinguistik kann dagegen jeweils fallweise auf Erkenntnisse der Grammatikforschung zu bestimmten Phänomenen aufbauen. Daneben findet selbstverständlich ein häufig sehr enger Dialog innerhalb bestimmter grammatiktheoretischer Forschungsansätze statt, insbesondere im Rahmen von Grammatikformalismen, für deren Entwicklung computerlinguistische Erwägungen mit verantwortlich waren, wie der Lexikalisch-Funktionale Grammatik (Kaplan/Bresnan 1982) und der Head-driven Phrase Structure Grammar (Pollard/Sag 1987, 1994).

Speziell aus Sicht der vergleichenden Grammatikforschung sind dabei Bestrebungen in der klassischen Computerlinguistik interessant, umfassende Computergrammatiken für mehrere Sprachen parallel nach einem sorgfältig abgestimmten Organisationsschema zu entwickeln. Im Rahmen des Formalismus der Lexikalisch-Funktionalen Grammatik wird dies seit Jahren im Rahmen des internationalen ParGram-Projekts betrieben (Butt et al. 2002); im Rahmen der Head-driven Phrase Structure Grammar gibt es einen ähnlichen Projektverbund namens DELPH-IN (Bender et al. 2002). Die Zielsetzung, nicht nur für beispielhafte Vergleichsphänomene die Gemeinsamkeiten und Unterschiede zwischen Grammatiken formal darstellbar zu machen, sondern praktikable Computergrammatiken mit breiter Phänomenabdeckung über Jahre hinweg kompatibel zu halten, erzwingt eine ganz spezielle sprach- 
vergleichende Herangehensweise, und es besteht ohne Zweifel ein großes Austauschpotenzial mit der traditionellen sprachvergleichenden Forschung, die zwar nicht komputationell orientiert ist, jedoch häufig ähnliche Anforderungen an die Breite der Phänomenabdeckung und die sprachübergreifende Vergleichbarkeit stellt.

Für eine exhaustive und theorieunabhängige automatische Korpusanalyse im Zuge der vergleichenden Grammatikforschung, wie ich sie ins Zentrum dieses Beitrags gestellt habe, bieten sich diese parallelen Grammatiken jedoch weniger an als die oben diskutierten statistischen Parser: die tiefen linguistischen Analysen sind relativ stark von den im Projektverbund entwickelten Annahmen geprägt (vgl. dazu die Diskussion in Abschnitt 2.1) und das Kriterium der linguistisch adäquaten Phänomenbehandlung überwiegt gegenüber einer robusten Abdeckung von beliebigen Eingaben.

Was die Unterstützung bei der studienübergreifenden automatischen Korpusannotation angeht, sind damit die Möglichkeiten, die aus der „klassischen" Computerlinguistik heraus kommen, trotz der vielfältigen anderweitigen Berührungspunkte beschränkt.

\subsection{Die „empirische Wende“ in der Computerlinguistik}

Nun haben, wie bereits erwähnt, die letzten 10-15 Jahre erhebliche Veränderungen im Selbstverständnis der Computerlinguistik mit sich gebracht. Der Einsatz von statistischen Verfahren zur Erfassung von systematischen Beziehungen in Korpusdaten hat sich zu einem zentralen Pfeiler der computerlinguistischen Forschung entwickelt. Schien die Forschungsgemeinde zu Anfang dieser Entwicklung in den 1990er Jahren noch in konkurrierende Lager gespalten, die jeweils mit ausschließlich statistischen bzw. ausschließlich symbolisch-linguistischen Methoden auszukommen versuchten, so besteht mittlerweile weitgehender Konsens darin, dass das Modellierungsziel für die Computerlinguistik den Erwerb von sprachlichem Wissen aus sprachlichem Input mit umfasst. In der systematischen Beschäftigung mit den eingesetzten Modellen und Formalismen rückt neben Fragen der Ausdrucksstärke und der Verarbeitungseigenschaften damit auch das Modellierungsziel der Lernbarkeit ins Zentrum des Interesses - also die Frage nach geeigneten maschinellen Lernverfahren für Probleme der Sprachverarbeitung. Mit modernen Verfahren lassen sich mittlerweile Analysewerkzeuge wie syntaktische Parser auf handannotierten Beispieldaten so gut „trainieren“, dass für die Anwendung bei der Analyse von neuen, aus dem Training nicht bekannten Sätzen eine erstaunliche Analysequalität erreicht werden kann - die Beispiele in Abschnitt 3.2 zum statistischen Dependenzparsing dürften einen Anhaltspunkt gegeben haben. 
Aufgrund des statistischen Trainings sind die Werkzeuge zudem insofern „robust“, als sie untypische Eingaben (beispielsweise sehr seltene Konstruktionen oder ungrammatische Sätze, wie sie je nach Sprachausschnitt in Korpusdaten durchaus häufiger vorkommen können) nicht zurückweisen, sondern für sie in aller Regel das nächstliegende kanonische Verhalten an den Tag legen - was in den meisten Anwendungsszenarien vorteilhaft ist. Ein offensichtlicher praktischer Vorteil gegenüber dem klassischen Ansatz liegt darüber hinaus darin, dass sich mit einer einmaligen Verbesserung des Trainingsverfahrens alle gleichartig trainierbaren Werkzeuge verbessern lassen, also insbesondere Werkzeuge für verschiedene Einzelsprachen - während in der klassischen Computerlinguistik die Entwicklung und Überarbeitung einer Grammatik für jede weitere Sprache einen erheblichen Aufwand darstellt.

Erkenntnisse aus der „klassischen“ Computerlinguistik fließen dabei zunehmend in eine gemischte Methodologie ein. Für bereits gut untersuchte Analyseprobleme lassen sich die besten Ergebnisse häufig dadurch erzielen, dass Erkenntnisse aus der Grammatikforschung für die Strukturierung des Problems (also die Definition der relevanten Repräsentationen) eingesetzt werden, auf die dann spezielle statistische Verfahren angewandt werden können.

Für die computerlinguistische Ausbildung hatte die methodologische Verlagerung in der Forschungslandschaft zur Konsequenz, dass neben der linguistischen Methodenkenntnis und den klassischen mathematischen Grundlagen im Bereich der formalen Logik, sowie Informatikkenntnissen für die algorithmische Umsetzung nun auch die mathematischen Grundlagen für Maschinelle Lernverfahren aus der Wahrscheinlichkeitstheorie und der Analysis benötigt werden: Das Trainieren eines computerlinguistischen Modells aufgrund von Korpusdaten stellt sich nun im allgemeinen Fall als Optimierungsproblem dar, z.B. müssen die Parameter eines Wahrscheinlichkeitsmodells so eingestellt werden, dass die Trainingsdaten maximal wahrscheinlich für das gewählte Modell werden.

\subsection{Grammatikforschung und „moderne“ Computerlinguistik}

Was bedeutet nun diese Schwerpunktverlagerung in der Computerlinguistik aus Sicht der einzelsprachlichen Sprachwissenschaften und insbesondere der sprachvergleichenden Grammatikforschung? Vordergründig scheint es die beiden sprachwissenschaftlichen Teildisziplinen zu entkoppeln. Fußte bislang die ,klassische“ computerlinguistische Forschung auf den gleichen formalen Grundlagen wie viele Arbeiten in der Grammatikforschung (wenn auch der Rückfluss von computerlinguistischen Ergebnissen nicht sehr ausgeprägt war), so scheint sich der linguistische Bezug in einem stark statistisch geprägten computerlinguistischen Ansatz auf die Annotation der Trainingsund Testdaten zu beschränken. Die trainierten Modelle können zwar, wie ge- 
schildert, ein sehr differenziertes und effektives Verhalten aufweisen, in dem grammatische Beschränkungen und außergrammatische statistische Tendenzen reflektiert sind - in den meisten Fällen kann man diese Zusammenhänge aber aus den Modellen nicht ablesen. Vielmehr ergibt sich das Verhalten aus dem Zusammenspiel einer Vielzahl von numerischen Parametern, die sich nur schwer auf einer symbolischen Ebene interpretieren lassen, so dass ein Rückfluss von Erkenntnissen in die Grammatikforschung noch wesentlich schwieriger erscheint. Beispielsweise könnte ein guter statistischer Parser, der lexikalische Merkmale berücksichtigt, aus den Trainingsdaten ein Verhalten abgeleitet haben, das die Subjekt-/Objektambiguität in $(17)^{28}$ korrekt auflöst.

(17) Über 7000 Brände müssen die Helfer bekämpfen.

Der Effekt wird jedoch aus dem Zusammenspiel von diversen Parametern entstehen: solchen zur Kombination von müssen und Brände bzw. Helfer als mögliche Subjekte (im Aktiv vs. im Passiv), entsprechend solchen für bekämpfen, daneben aber auch Parametern zur generellen Tendenz, Akkusativobjekte im Vorfeld zu platzieren und der Tendenz, dass Brände als Objekt fungiert (unabhängig vom Verb) usw. Eine einzelne ausschlaggebende Beschränkung (beispielsweise, dass bekämpfen einen belebten Agens verlangt) wird man üblicherweise nicht isolieren können - vor allem nicht in subtileren Fällen.

Vordergründig könnte folgender Eindruck entstehen: Die Methoden der modernen, statistischen Computerlinguistik führen zwar innerhalb der Computerlinguistik zu interessanten Entwicklungsmöglichkeiten, in Ergänzung zur klassisch symbolischen Modellierung (und diese Möglichkeiten werden auch in der Tat sehr erfolgreich exploriert) - die Methoden scheinen jedoch für die sprachwissenschaftlichen Disziplinen ,jenseits“ der symbolischen Computerlinguistik, denen es um eine Beschreibung und Erklärung der Regularitäten in der Grammatik einer oder mehrerer Sprachen geht, nur sehr mittelbar von Belang zu sein.

Die Schlussfolgerung wäre also, dass die moderne Computerlinguistik die gemeinsame Basis mit der Grammatikforschung verloren hätte. Gab (und gibt) es mit der „klassischen“ Computerlinguistik trotz unterschiedlicher methodologischer Herangehensweisen deutliche Berührungspunkte im Ziel der Untersuchungen - eine präzise symbolische Beschreibung des grammatischen Systems -, so hat sich die statistisch geprägte Computerlinguistik mit der Einführung von relativ opaken Modellen aus dem Maschinellen Lernen von diesem Berührungspunkt entfernt.

Gerade in Bezug auf die Frage der Methodenkompetenz für die künftige Ausbildung für die Grammatikforschung hätte eine solche Schlussfolgerung natürlich erhebliche Auswirkungen.

28 Internet-Beleg (02.08.2010): www.n-tv.de/panorama/Medwedew-ruft-Notstand-aus-article1186016.html. 


\section{Ausblick und Konklusion}

Der im letzten Abschnitt geschilderte vordergründige Eindruck einer langfristigen Divergenzbewegung zwischen Grammatikforschung und Computerlinguistik erklärt sicherlich, warum es auf der Ebene der Modellierung derzeit nur wenige ausgeprägte Kooperationen gibt, und er könnte dazu führen, dass in der Ausbildung für die Grammatikforschung auf eine tiefere Beschäftigung mit modernen computerlinguistischen Methoden weiter verzichtet wird (abseits der Überlegung, ein anwendungsorientiertes „zweites Standbein" anzubieten). Ich halte die angedeutete Schlussfolgerung jedoch für falsch, und zwar auf zwei Ebenen.

Zum ersten, und das sollte die ausführliche Illustration in Abschnitt 3 untermauern, sind die statistischen Verfahren mittlerweile bei einer Qualitätsstufe angelangt, die automatische Verfahren jenseits des Part-of-SpeechTaggings als ein Werkzeug (neben anderen traditionelleren Werkzeugen) für die Grammatikforschung attraktiv machen dürfte - zumal Korpusuntersuchungen in der Grammatikforschung mehr und mehr Gewicht zukommt. Die automatischen Analysewerkzeuge können den Daten-Explorationsprozess bei der Untersuchung von vielen seltenen Konstruktionen erheblich erleichtern. Gerade die sprachvergleichende Forschungsarbeit kann bei der Parallel-Exploration in zwei oder mehr Sprachen von der Verfügbarkeit von gleichartigen Grammatikanalysewerkzeugen profitieren und möglicherweise mittels automatischer Wortalignierung (wie in Abschnitt 3.3 dargestellt) den Blick auf unerwartete Konfigurationen in einem sehr großen Parallelkorpus lenken, wie sie mit traditionellen Mitteln nur durch Zufall zu entdecken sind.

Allerdings sind die Werkzeuge - wie die Darstellung in diesem Beitrag schon andeutungsweise gezeigt hat - zumeist Ergebnis eines verhältnismäBig komplexen Konstruktionsprozesses, und entsprechend geht die Analyse nicht so vor sich, wie das für einen menschlichen Benutzer mit seinen Annahmen zu grammatischen Regularitäten und Beschränkungen einerseits und seinem inhaltlichen, kontextgebundenen Verständnis andererseits zu erwarten ist. Selbst ein sehr gutes Werkzeug wird Fehler an Stellen machen, die bei einem menschlichen Annotator nicht zu erwarten sind (und andererseits wird es bestimmte Arten von unsystematischen Fehlern nicht geben). Um ein derartiges Hilfsmittel also optimal einsetzen zu können, ist ein Grundverständnis zu seiner Arbeitsweise sicherlich ausgesprochen hilfreich - genauso wie in anderen empirischen Wissenschaften die Eigenheiten der verwendeten Instrumente, wie etwa Mikroskope oder Teleskope unterschiedlicher Funktionsweisen, genau bekannt sein müssen.

Dies sollte selbstverständlich soweit wie möglich durch die Computerlinguistik unterstützt werden. Eine Initiative zu diesem Themenkomplex ist das europaweite Infrastruktur-Projekt CLARIN (Common Language Resources and Technology Infrastructure), in dem Sprachressourcen und -werkzeuge spe- 
ziell für die geisteswissenschaftliche Forschung in leicht zugänglicher und nachhaltig verfügbarer Form bereitgestellt werden. In Deutschland beteiligen sich mehrere Zentren an dem durch das Bundesministerium für Bildung und Forschung geförderten Verbundprojekt CLARIN-D. Gerade für zukünftige Generationen von Grammatikforschern wird hoffentlich der Zugang zu den verfügbaren Werkzeugen eine Selbstverständlichkeit werden.

Ich möchte jedoch abschließend zusätzlich für eine noch anspruchsvollere Ebene der Kooperation argumentieren: Über den einseitigen Informationsfluss von der statistischen Computerlinguistik hin zur Grammatikforschung (als reine Anwenderin von vorgegebenen automatischen Werkzeugen für die Korpusarbeit) hinaus besteht das Potenzial für eine tiefere, organischere Verbindung der beiden sprachwissenschaftlichen Disziplinen - und das vielleicht ganz besonders, wenn es um sprachvergleichende Arbeiten geht. Je ausgereifter die statistischen Verfahren werden, umso stärkeres Gewicht kommt auch feingliedrigen Unterscheidungen zu, wenn es darum geht, die Qualität der Analysekomponenten noch weiter zu verbessern. Hier ist häufig feiner linguistischer Sachverstand gefragt. Dieser lässt sich auf der Ebene der üblichen, generischen Analysewerkzeuge sicherlich durch linguistisch gut ausgebildete Computerlinguistinnen und Computerlinguisten einbringen - für die Verbesserung der Analysewerkzeuge, wie sie speziell im Rahmen einer linguistischen Korpusuntersuchung zum Einsatz kommen, wäre es jedoch am effektivsten, wenn die Trennung zwischen den Werkzeugentwicklern und den Anwendern aufgeweicht und eine interaktive Anpassung der relevanten Aspekte der Analyse ermöglicht werden könnte.

Konkret auf das Beispiel in Abschnitt 3 bezogen: Durch eine sukzessive Verbesserung der Analysequalität beim Parsing und bei der Wortalignierung durch die Entwickler können die Werkzeuganwender sicherlich erwarten, dass der eine oder andere Fehler in der generischen Analyse bei einer zukünftigen, verbesserten Werkzeugversion vermieden wird (beispielsweise werden Subjekte noch zuverlässiger erkannt). Damit wird sich hoffentlich auch die Effektivität des Werkzeugeinsatzes verbessern.

Die generischen Aspekte der Analyse (die Erkennung von Subjekten, Relativsätzen etc. im Deutschen und im Englischen) werden jedoch bei einem spezifischen Einsatz in der (vergleichenden) Grammatikforschung auf eine ganz bestimmte Art kombiniert (beispielsweise durch syntaktische Templates für Spaltsatzkonstruktionen, die mittels einer Wortalignierung kontrastiv in Beziehung gesetzt werden). Durch die Kombination der Aspekte entstehen komplexe Strukturkonfigurationen, zu denen sich selbstverständlich weder die Werkzeugentwickler noch die Annotatoren der zugrunde liegenden Trainingsdaten Gedanken gemacht haben. Mit dem speziellen Wissen der Grammatikforscherin oder des Grammatikforschers ließen sich für die komplexen Konfigurationen mit verhältnismäßig geringem Aufwand Verfeinerungen der automatischen Analyse vornehmen, von denen zumin- 
dest die jeweilige empirische Studie profitieren könnte - im günstigen Fall sogar spätere Studien, die indirekt darauf aufbauen. (Ein denkbares Beispiel für eine konfigurationsspezifische Optimierung wäre die Ausnutzung der Wortalignierung des Expletivpronomens in der Spaltsatzkonstruktion mit klar referenziellen Pronomina in einer parallelen Sprache für das Training eines speziellen Spaltsatz-Klassifikators mit einer erhöhten Precision (vgl. dazu Fußnote 11). Der Weg über eine oder mehrere Kontrastsprachen in einem Parallelkorpus ist stets ein vielversprechender Weg, um ein unabhängiges Korrektiv einzubinden, ohne dass eine umfassende manuelle Annotation nötig wird.)

Um derartige Schritte zu ermöglichen, sind natürlich mehrere Entwicklungen nötig: Zum einen müssen die Werkzeuge in einer trainierbaren Form angeboten werden, und es muss eine Arbeitsumgebung geben, die einen solchen Prozess unterstützt. Zum anderen sind jedoch ein Verständnis zur grundsätzlichen Arbeitsweise von Maschinellen Lernverfahren und eine möglichst große praktische Erfahrung seitens der Grammatikforscher erforderlich, damit innerhalb eines großen Raums von Möglichkeiten diejenigen Anpassungen vorgenommen werden können, die am ehesten erfolgversprechend sind.

Dies würde freilich völlig entgegen der hypothetischen Schlussfolgerung am Ende von Abschnitt 4 dafür sprechen, der Auseinandersetzung mit „modernen“ computerlinguistischen Methoden in der Ausbildung für die Grammatikforschung großes Gewicht zu geben.

Was vielleicht wie der Vorschlag für eine sehr radikale Wendung im Verhältnis zwischen Grammatikforschung und Computerlinguistik klingt, ist im Grunde genommen nur das konsequente Zusammenführen der zwei wesentlichen Wurzeln für die moderne Computerlinguistik: In der Sprachtechnologie und statistischen Computerlinguistik wird von jeher mit Datensammlungen gearbeitet, die aus den akkumulierten Erkenntnissen von Jahrzehnten von deskriptiver und theoretisch geprägter linguistischer Forschungsarbeit hervorgegangen sind. Die Baumbanken, auf denen die in Abschnitt 3 dargestellten Parser trainiert werden (und die teilweise in Abschnitt 2 aufgelistet sind), wurden anhand von Annotationsrichtlinien annotiert, in denen die Erkenntnisse aus der Grammatikforschung kondensiert wurden. Bis heute werden jedoch in den allermeisten Fällen die Trainingsdaten für statistische Analysewerkzeuge als eine gegebene, statische Menge betrachtet, auf deren Grundlage eine möglichst hohe Analysequalität erzielt werden soll - ohne dass linguistische Annotatoren und die Spezialisten für Maschinelles Lernen jemals in einen direkten Austausch treten. ${ }^{29}$ Niemand würde in Zweifel ziehen, dass die kompetent vorgenommene Anpassung

$29 \quad$ Eine Ausnahme stellt der Ansatz des Active Learning dar, bei dem die Annotationsarbeit und das Training der Werkzeuge so miteinander verschränkt werden, dass die Auswahl der jeweils als nächstes zu annotierenden Daten laufend aufgrund von Eigenschaften des bis dahin 
der ursprünglichen Annahmen in einer Sprachressource große Chancen für eine verbesserte Analysequalität der darauf aufbauenden Werkzeuge bietet. Und der Grund dafür, dass derartige Schritte nicht häufiger vorgenommen werden, scheint mir vor allem in der Trennung der ,zuständigen“ Teildisziplinen und dem jeweiligen traditionellen Methodenkanon zu liegen. Dabei ist der (vergleichenden) Grammatikforschung und der modernen Computerlinguistik das Bestreben gemein, die ganze Breite der grammatischen Ausdrucksformen zu erfassen und dabei auch feine Unterschiede in einer angemessenen Form repräsentationell zu verankern und klassifikatorisch auseinanderzuhalten.

Die in Abschnitt 4 beobachtete Divergenz ist also aus dem Gegenstand heraus nicht motiviert, sondern scheint vordringlich einen - sicherlich nicht einfach zu überwindenden - methodologischen Kern zu haben. Es dürfte sich also lohnen, mit den jeweiligen Methoden aufeinander zuzugehen, um die Trennung zu überwinden. Selbstverständlich lässt sich dies nicht von heute auf morgen und in einem einzigen, vorspezifizierten Projekt umsetzen. Im Gegenteil: damit größere Teile der unterschiedlichen Fachcommunities ,mitgenommen“ werden können, sollte es sich um einen länger angelegten Prozess handeln.

Ich halte diese Überlegung jedoch für ein sehr starkes Argument für interdisziplinäre Verbundforschung wie beispielsweise linguistische/computerlinguistische Sonderforschungsbereiche, in denen ausgewiesene Spezialisten aus unterschiedlichen methodologischen Traditionen versuchen, den etablierten disziplininternen Erfolgskriterien genüge zu tun und gleichzeitig neue Wege der Methodenkombination zu explorieren.

\section{Danksagung}

Die Arbeiten, die in Abschnitt 3 teilweise referiert und in den Abbildungen dargestellt wurden, wurden im Rahmen des Sonderforschungsbereichs 632 an der Universität Potsdam (insbesondere in Teilprojekt D4) und im Rahmen des Sonderforschungsbereichs 732 an der Universität Stuttgart (insbesondere in Teilprojekt D8) von der Deutschen Forschungsgemeinschaft gefördert. Meine jeweiligen Koautoren hatten erheblichen Anteil an den Forschungsergebnissen. Der Aufbau eines CLARIN-D-Zentrums für Sprachanalysewerkzeuge am Institut für Maschinelle Sprachverarbeitung der Uni-

trainierten Systems bestimmt werden: Aufgrund einer Selbsteinschätzung der Konfidenz des Systems bei der Analyse von unbekannten Daten werden diejenigen Instanzen ermittelt, deren Annotation für besonders informativ erachtet wird.

Derartige Verfahren werden in Simulationsstudien relativ stark diskutiert, kommen jedoch in der (akademischen) Praxis der Sprachanalyse bislang nicht in größerem Maßstab zum Einsatz. 
versität Stuttgart wird durch das Bundesministerium für Bildung und Forschung im Rahmen des CLARIN-D-Verbundprojekts gefördert; eine zusätzliche Förderung erfolgt durch das Ministerium für Wissenschaft, Forschung und Kunst Baden-Württemberg.

Ich danke den Veranstaltern der IDS-Jahrestagung für die Gelegenheit, einen Beitrag aus Sicht der Computerlinguistik zu leisten, und zwei anonymen Gutachtern für sehr wertvolle Kommentare zu diesem Beitrag.

\section{Literatur}

Belica, Cyril et al. (2011): The Morphosyntactic Annotation of DeReKo: Interpretation, Opportunities, and Pitfalls. In: Konopka, Marek et al. (Hg.): Grammatik und Korpora 2009. Dritte Internationale Konferenz. Mannheim, 22.4.-24.9.2009. (= Korpuslinguistik und interdisziplinäre Perspektiven auf Sprache 1). Tübingen, S. 451-469.

Bender, Emily M./Flickinger, Dan/Oepen, Stephan (2002): The grammar Matrix. An open-source starter-kit for the rapid development of cross-linguistically consistent broad-coverage precision grammar. In: Proceedings of the Workshop on Grammar Engineering and Evaluation at the 19th International Conference on Computational Linguistics. Taipei, S. 8-14.

Bohnet, Bernd (2010): Top accuracy and fast dependency parsing is not a contradiction. In: Proceedings of the 23rd International Conference on Computational Linguistics (COLING 2010). Peking, S. 89-97.

Bouma, Gerlof/Øvrelid, Lilja/Kuhn, Jonas (2010): Towards a large parallel corpus of cleft constructions. In: Proceedings of the Seventh Conference on International Language Resources and Evaluation (LREC'10). Valletta, S. 3585-3592.

Brown, Peter F. et al. (1990): A statistical approach to machine translation. In: Computational Linguistics 6, 2, S. 79-85.

Brown, Peter F. et al. (1993): The mathematics of statistical machine translation: Parameter estimation. In: Computational Linguistics 19, 2, S. 263-311.

Butt, Miriam et al. (2002): The Parallel Grammar Project. In: Proceedings of COLING2002 Workshop on Grammar Engineering and Evaluation. Taipei, S. 1-7.

Christ, Oliver (1994): A modular and flexible architecture for an integrated corpus query system. In: Proceedings of the 3rd Conference on Computational Lexicography and Text Research (COMPLEX'94). Budapest, S. 23-32.

Dione, Cheikh M.B./Kuhn, Jonas/Zarrieß, Sina (2010): Design and development of part-of-speech-tagging resources for Wolof (Niger-Congo, spoken in Senegal). In: Proceedings of the Seventh conference on International Language Resources and Evaluation (LREC'10). Valletta, S. 2806-2813.

Dufter, Andreas (2009): Clefting and discourse organization: comparing Germanic and Romance. In: Dufter, Andreas/Jacob, Daniel (Hg.): Focus and Background in Romance Languages. (= Studies in Language Companion Series 112). Amsterdam u.a., S. 83-121. 
Gale, William A./Church, Kenneth W. (1993): A program for aligning sentences in bilingual corpora. In: Computational Linguistics 19, 1 (Special Issue on Using Large Corpora), S. 75-102.

Gast, Volker/Wiechmann, Daniel (i.d.Bd.): $W(b)$-Clefts im Deutschen und Englischen: Eine quantitative Untersuchung auf Grundlage des Europarl-Korpus.

Giesbrecht, Eugenie/Evert, Stefan (2009): Part-of-speech tagging - a solved task? An evaluation of POS taggers for the Web as corpus. In: Alegria, Inaki/Leturia, Igor/ Sharoff, Serge (Hg.): Proceedings of the 5th Web as Corpus Workshop (WAC5). San Sebastian, S. 27-35.

Johansson, Mats (2001): Clefts in contrast: a contrastive study of clefts and $w h$ clefts in English and Swedish texts and translations. In: Linguistics 39, 3, S. 547-582.

Kaplan, Ronald M./Bresnan, Joan (1982): Lexical-functional grammar: a formal system for grammatical representation. In: Bresnan; Joan (Hg.): The mental representation of grammatical relations. Cambridge, MA.

Kayne, Richard (1975): French syntax: The transformational cycle. Cambridge, MA.

Koehn, Philipp (2005): Europarl: A parallel corpus for statistical machine translation. In: Proceedings of the Tenth Machine Translation Summit (MT Summit X). Phuket, S. 79-86.

Meurers, Detmar/Müller, Stefan (2009): Corpora and Syntax. In: Lüdeling, Anke/Kytö, Merja (Hg.): Corpus Linguistics. Bd. 2, Kapitel 42. (= Handbücher zur Sprach- und Kommunikationswissenschaft 29.2). Berlin, S. 920-933.

Nivre, Joakim/Hall, Johan/Nilsson, Jens (2006a): Maltparser: a data-driven parser-generator for dependency parsing. In: Proceedings of the Fifth International Conference on Language Resources and Evaluation (LREC). Genua, S. 2216-2219.

Nivre, Joakim/Nilsson, Jens/Hall, Johan (2006b): Talbanken05: a Swedish treebank with phrase structure and dependency annotation. In: Proceedings of the Fifth International Conference on Language Resources and Evaluation (LREC). Genua, S. 1392-1395.

Pollard, Carl/Sag, Ivan A. (1987): Information-based syntax and semantics. Bd. 1: Fundamentals. (= CSLI Lecture Notes 13). Stanford.

Pollard, Carl/Sag, Ivan A. (1994): Head-Driven Phrase Structure Grammar. Chicago.

Seeker, Wolfgang et al. (2010a): Informed ways of improving data-driven dependency parsing for German. In: Proceedings of the 23rd International Conference on Computational Linguistics (COLING 2010): Poster Volume. Peking, S. 1122-1130.

Seeker, Wolfgang et al. (2010b): Hard constraints for grammatical function labelling. In: Proceedings of the 48th Annual Meeting of the Association for Computational Linguistics (ACL 2010). Uppsala, S. 1087-1097.

Seeker, Wolfgang/Kuhn, Jonas (2011): On the role of explicit morphological feature representation in syntactic dependency parsing for German. In: Proceedings of IWPT 2011, 12th International Conference on Parsing Technologies. Dublin, S. 58-62. 
Tiedemann, Jörg/Nygaard, Lars (2004): The OPUS corpus - parallel and free. In: Proceedings of the Fourth International Conference on Language Resources and Evaluation (LREC'04). Bd. 4. Lissabon, S. 1183-1186.

van Noord, Gertjan (2006): At last parsing is now operational. In: Mertens, Piet et al. (Hg.): Verbum Ex Machina. Actes de la 13e Conference sur le Traitement Automatique des Langues Naturelles (TALN 2006). (= Cahiers du Cental 2). Louvain-laNeuve, S. 20-42.

Yarowsky, David/Ngai, Grace/Wicentowski, R. (2001): Inducing multilingual text analysis tools via robust projection across aligned corpora. In: Proceedings of the Human Language Technology Conference (HLT-2001), San Diego, S. 161-168.

Yuret, Deniz/Han, Aydın/Turgut, Zehra (2010): SemEval-2010 Task 12: Parser Evaluation using Textual Entailments. In: Proceedings of the 5th International Workshop on Semantic Evaluation, Association for Computational Linguistics. Uppsala, S. 51-56. 